\title{
POSSIBLE CARDINALITIES OF MAXIMAL ABELIAN SUBGROUPS OF QUOTIENTS OF PERMUTATION GROUPS OF THE INTEGERS
}

\author{
SAHARON SHELAH AND JURIS STEPRĀNS
}

\section{InTRODUCTION AND DEFINITIONS}

The maximality of Abelian subgroups play a role in various parts of group theory. For example, Mycielski [8, 7] has extended a classical result of Lie groups and shown that a maximal Abelian subgroup of a compact connected group is connected and, furthermore, all the maximal Abelian subgroups are conjugate. For finite symmetric groups the question of the size of maximal Abelian subgroups has been examined by Burns and Goldsmith in 国 and Winkler in 15 . It will be shown in Corollary 3.1 that there is not much interest in generalizing this study to infinite symmetric groups; the cardinality of any maximal Abelian subgroup of the symmetric group of the integers is $2^{\aleph_{0}}$. The purpose of this paper is to examine the size of maximal Abelian subgroups for a class of groups closely related to the the symmetric group of the integers; these arise by taking an ideal on the integers, considering the subgroup of all permutations which respect the ideal and then taking the quotient by the normal subgroup of permutations which fix all integers except a set in the ideal. It will be shown that the maximal size of Abelian subgroups in such groups is sensitive to the nature of the ideal as well as various set theoretic hypotheses.

The reader familiar with applications of the Axiom of Choice may not be surprised by the assertion just made since, one can imagine constructing ideals on the integers by transfinite induction such that the quotient group just described exhibits various desired properties. Consequently, it is of interest to restrict attention to only those ideals which do not require the Axiom of Choice for their definition. All of the ideals considered will here will have simple definitions - indeed, they will all be Borel subsets of $\mathcal{P}(\omega)$ with the usual topology - and, in fact, the first three sections will focus on the ideal of finite sets. It should be mentioned that there is large body of work examining the analogous quotients of the Boolean algebra $\mathcal{P}(\omega)$ modulo an analytic ideal - the monograph [5] by Farah is a good reference for this subject. However, the analogy is far from perfect since, for example, whereas the Boolean algebra $\mathcal{P}(\omega) /[\omega]]^{<\aleph_{0}}$ can consistently have $2^{2^{\aleph_{0}}}$ automorphisms [9] it is shown in [1] that the quotient of the full symmetric group of the integers modulo the subgroup of finite permutations has only countably many outer automorphisms. Nevertheless, it may be possible to employ methods similar to those of 5 in order to distinguish between different quotient algebras up to isomorphism. This has been done for elementary equivalence in [11, 13] for quotients of the full symmetric group on $\kappa$ by the normal subgroups fixing all but $\lambda$ elements. However since the full symmetric group of the integers has only two proper normal subgroups [10] quotients of certain naturally arising subgroups will be considered instead. One of the goals of this study is to use the cardinal invariant associated with maximal Abelian subgroups as a tool to distinguish between isomorphism types of such groups.

In order to state the main results precisely some notation is needed.

Definition 1.1. If $G$ is a group then define the Abelian subgroup spectrum of $G$ to be the set of all $\kappa$ such that there is a maximal Abelian subgroup of $G$ of size $\kappa$. Define $A(G)$ to be least uncountable cardinal in the Abelian subgroup spectrum of $G$.

Notation 1.1. Through this paper the symbol $\mathbb{S}$ will be used to denote the symmetric group on $\mathbb{N}$. For $\pi \in \mathbb{S}$ let $\operatorname{supp}(\pi)$ denote the support of $\pi$ which is defined to be $\{x \in \operatorname{domain}(\pi): \pi(x) \neq x\}$. If

1991 Mathematics Subject Classification. 03E17, 03E35, 03E40, 03E50, 20B07, 20B30, $20 \mathrm{~B} 35$.

The research of the first author was supported by The Israel Science Foundation founded by the Israel Academy of Sciences and Humanities, and by NSF grant No. NSF-DMS97-04477. Research of the second author for this paper was partially supported by NSERC of Canada. This is number 786 on the first author's personal list of publications. 
$\mathcal{I}$ is an ideal] on $\mathbb{N}$ then $\mathbb{S}(\mathcal{I}) \subseteq \mathbb{S}$ will denote the subgroup of all permutations preserving $\mathcal{I}$; in other words, a permutation $\pi$ belongs to $\mathbb{S}(\mathcal{I})$ provided $\pi(A) \in \mathcal{I}$ if and only if $A \in \mathcal{I}$. On the other hand, $\mathbb{F}(\mathcal{I})$ will be used to denote the normal subgroup of $\mathbb{S}(\mathcal{I})$ consisting of all permutations $\pi \in \mathbb{S}(\mathcal{I})$ such that $\operatorname{supp}(\pi) \in \mathcal{I}$. The abbreviation $\mathbb{F}=\mathbb{F}\left([\mathbb{N}]<\aleph_{0}\right.$ will also be used.

The focus of this paper will be on examining computing $A(\mathbb{S}(\mathcal{I}) / \mathbb{F}(\mathcal{I}))$ for various simply defined ideals. This cardinal will be denoted by $A(\mathcal{I})$.

Notation 1.2. Given a pair of permutations $\left\{\pi, \pi^{\prime}\right\} \in[\mathbb{S}]^{2}$ define $\operatorname{NC}\left(\pi, \pi^{\prime}\right)=\left\{n \in \mathbb{N}: \pi\left(\pi^{\prime}(n)\right) \neq\right.$ $\left.\pi^{\prime}(\pi(n))\right\}$. A pair of permutations $\left\{\pi, \pi^{\prime}\right\} \in[\mathbb{S}]^{2}$ will be said to almost commute modulo an ideal $\mathcal{I}$ if $\operatorname{NC}\left(\pi, \pi^{\prime}\right) \in \mathcal{I}$ and they will be said to almost commute if $\operatorname{NC}\left(\pi, \pi^{\prime}\right)$ is finite.

Notation 1.3. Given a permutation $\pi$ and a $X \subseteq \mathbb{N}$ define the orbit of $X$ under $\pi$ by $\operatorname{orb}_{\pi}(X)=$ $\left\{\pi^{i}(x)\right\}_{i \in \mathbb{Z}, x \in X}$. The abbreviation $\operatorname{orb}_{\pi}(n)=\operatorname{orb}_{\pi}(\{n\})$ will be used when no confusion is possible.

Notation 1.4. Given a permutation $\pi \in \mathbb{S}$ define $\equiv_{\pi}$ be the equivalence relation on $\mathbb{N}$ whose equivalence classes are the orbits of $\pi$. Given a set of permutations $\mathcal{S} \subseteq \mathbb{S}$ define $\equiv_{\mathcal{S}}$ to be the transitive closure of the set of equivalence relations $\left\{\equiv_{\pi}\right\}_{\pi \in \mathcal{S}}$. Let $\Omega_{\mathcal{S}}$ denote the set of equivalence classes of $\equiv_{\mathcal{S}}$ and, for any set $X$ define $\Omega_{\mathcal{S}}(X)$ to be the smallest set containing $X$ and closed under equivalence classes of $\equiv \mathcal{S}$.

Notation 1.5. Given two finite subsets $A$ and $B$ of $\mathbb{N}$ define $\Delta_{A, B}: A \rightarrow B$ to be the unique order preserving mapping between them and let $\Delta_{A}=\Delta_{A,|A|}$. If $\mathcal{S} \subseteq \mathbb{S}$ and $A$ and $B$ are two equivalence classes of $\equiv_{\mathcal{S}}$ then define $A$ to be $\mathcal{S}$-isomorphic to $B$ if there is a bijection $\psi: A \rightarrow B$ such that $\pi(\psi(a))=\psi(\pi(a))$ for each $\pi \in \mathcal{S}$ and $a \in A$.

The set theoretic notation used throughout will follow the contemporary standard. In particular, $[X]^{k}$ will denote the family of subsets of $X$ of cardinality $k$ and $[X]^{<k}$ will denote the family of subsets of $X$ of cardinality less than $k$. Cardinal invariants of the continuum are closely linked to investigation of $A(\mathcal{I})$. The following recall the definitions of some well known invariants.

Definition 1.2. Given an ideal $\mathcal{I} \subseteq \mathcal{P}(\omega)$ let $\mathcal{P}(\omega) / \mathcal{I}$ be the quotient Boolean algebra and denote the least cardinal of a maximal, uncountable, pairwise disjoint family In the special case $\mathcal{I}=[\mathbb{N}]^{<\aleph_{0}} \mathfrak{a}(\mathcal{I})$ is denoted by $\mathfrak{a}$ The least cardinal of an ideal $\mathcal{B} \subseteq \mathcal{P}(\omega) /[\omega]<\aleph_{0}$ such that there is no $C \in \mathcal{P}(\omega) /[\omega]^{<\aleph_{0}}$ disjoint from all members of $\mathcal{B}$ other than the equivalence class of the finite sets is denoted by $\mathfrak{p}$.

In Section 2 it is shown that $\mathfrak{a}$ is an upper bound for $A\left([\mathbb{N}]^{<\aleph_{0}}\right)$ while in Section 3 it is shown that $\mathfrak{p}$ serves as a lower bound for $A\left([\mathbb{N}]^{<\aleph_{0}}\right)$. Sections 4 and 5 deal with consistency results. In Section 4 it is shown that $\mathfrak{a}$ is not the best possible upper bound for $A\left([\mathbb{N}]^{<\aleph_{0}}\right)$ since in the iterated Laver model $A\left([\mathbb{N}]^{<\aleph_{0}}\right)$ is strictly less than $\mathfrak{a}$. Sections 5 and 6 deal with quotients using ideals other than the ideal of finite sets. It is shown in Section 5 that adding $\aleph_{1}$ Cohen reals to a model where $2^{\aleph_{0}}>\aleph_{1}$ yields a model where $A\left(\mathcal{I}_{1 / x}\right)=\aleph_{1}<2^{\aleph_{0}}$ and $\mathcal{I}_{1 / x}$ is the ideal of sets whose reciprocals form a series with finite sum. Section 6 deals with ideals similar to the density ideal. It is shown that $A(\mathcal{I})=2^{\aleph_{0}}$ for many of these ideals $\mathcal{I}$. No extra set theoretic axioms are used here. The final section contains some open questions.

\section{AN UPPER BOUND}

Proposition 2.1. $A\left([\mathbb{N}]^{<\aleph_{0}}\right) \leq \mathfrak{a}$.

Proof. Let $\mathcal{A}$ be a maximal almost disjoint family of subsets of $\mathbb{N}$ of size $\mathfrak{a}$ and let $F(\mathcal{A})$ be the free Abelian group generated by $\mathcal{A}$ under coordinate wise addition; in other words, if $f \in F(\mathcal{A})$ then $f: \mathcal{A} \rightarrow \mathbb{Z}$ and $f$ has finite support. For $a \in \mathcal{A}$ define $\pi_{a}: a \rightarrow a$ by $\pi_{a}(i)=\min (\{j \in a: j>i\})$ and, for $j \in \mathbb{Z}$, let $\pi_{a}^{j}$ denoted the $j$-fold composition of $\pi_{a}$ noting that both the domain and range

\footnotetext{
${ }^{1}$ An ideal is a collection of subsets of the integers closed under finite unions and subsets.

${ }^{2}$ See [14] for a more detailed discussion of this invariant.
} 
of $\pi_{a}^{j}$ are co-finite subsets of $a$.. If $f \in F(\mathcal{A})$ then let $\Phi(f)$ be the set of all permutations $\pi$ such that there is a finite set $F \subseteq \mathbb{N}$ such that

$$
\pi(j)= \begin{cases}\pi_{a}^{f(a)}(j) & \text { if } j \in a \backslash F \text { and } f(a) \neq 0 \\ j & \text { if } j \notin F \text { and }(\forall a \in \mathcal{A}) j \notin a \text { or } f(a)=0\end{cases}
$$

leaving $\Phi(f)$ undefined if there are no such permutations. Note that if $\pi \in \Phi(f)$ and $\sigma \in \Phi(g)$ then $\pi \circ \theta \in \Phi(f+g)$. Since $\Phi$ is easily seen to be one-to-one, it is an isomorphism between a subgroup of $F(\mathcal{A})$ and the subgroup $\Phi(F(\mathcal{A}))$ of $\mathbb{S} / \mathbb{F}$.

In fact, $\Phi(f)$ is defined precisely when $\sum_{a \in \mathcal{A}} f(a)=0$. To see this, let $f \in F(\mathcal{A})$ and suppose that the support of $f$ is $B$ and $\sum_{b \in B} f(b)=0$. Let $F \subseteq \mathbb{N}$ be a finite set such that $b \cap b^{\prime} \subseteq F$ for any two $b$ and $b^{\prime}$ in $B$ and such that $F \cap b$ is an initial segment of $b$ for each $b \in B$. Let $B^{+}=\{b \in B: f(b)>0\}$ and $B^{-}=\{b \in B: f(b)<0\}$. If $b \in B^{+}$let $b^{*}$ be the first $f(b)$ elements of $b \backslash F$ and if $b \in B^{-}$let $b^{*}$ be the first $-f(b)$ elements of $b \backslash F$. Let $\theta: \bigcup_{b \in B^{-}} b^{*} \rightarrow \bigcup_{b \in B^{+}} b^{*}$ be any bijection and define $\pi$ as follows:

$$
\pi(j)= \begin{cases}j & \text { if } j \notin \bigcup_{b \in B} b \backslash F \\ \pi_{b}^{f(b)} & \text { if } j \in b \in B^{+} \\ \pi_{b}^{f(b)} & \text { if } j \in b \backslash b^{*} \text { and } b \in B^{-} \\ \theta(j) & \text { if } j \in \bigcup_{b \in B^{-}} b^{*}\end{cases}
$$

and observe that $\pi$ is a bijection.

To see that $\Phi(F(\mathcal{A}))$ is maximal Abelian let $[\pi]_{\mathbb{F}} \in \mathbb{S} / \mathbb{F} \backslash \Phi(F(\mathcal{A}))$. Before continuing some notation will be introduced. Given two distinct elements $a$ and $a^{\prime}$ of $\mathcal{A}$ define $f_{a, a^{\prime}} \in F(\mathcal{A})$ be such that $\operatorname{supp}\left(f_{a, a^{\prime}}\right)=\left\{a, a^{\prime}\right\}$ and $f_{a, a^{\prime}}(a)=1=-f_{a, a^{\prime}}\left(a^{\prime}\right)$. Choose $\pi_{a, a^{\prime}} \in \Phi\left(f_{a, a^{\prime}}\right)$.

Claim 1. If $a \in \mathcal{A}$ is such that $\operatorname{supp}(\pi) \cap a$ is infinite then $\operatorname{supp}(\pi) \cap a$ is a co-finite subset of $a$.

Proof. Let $a^{\prime} \in \mathcal{A} \backslash\{a\}$ If the claim fails then there are infinitely many $n \notin a$ such that $n \in \operatorname{supp}(\pi)$ but $\pi_{a, a^{\prime}}(n) \in \operatorname{supp}(\pi)$. For any such $n$ it follows that $\pi \circ \pi_{a, a^{\prime}}(n) \neq \pi_{a, a^{\prime}}(n)$ while $\pi_{a, a^{\prime}} \circ \pi(n)=\pi_{a, a^{\prime}}(n)$. Hence $[p i]_{\mathbb{F}}$ and $\left[p i_{a, a^{\prime}}\right]_{\mathbb{F}}$ do not commute.

Claim 2. If $a \in \mathcal{A}$ is such that $\operatorname{supp}(\pi) \cap a$ is infinite then $\pi(a) \subseteq^{*} a$.

Proof. If not, there are infinitely many $n \in a$ such that $\pi(n) \notin a$. Let $X$ be the set of all such $n$ and choose $a^{\prime} \in \mathcal{A}$ such that $\pi(X) \backslash a^{\prime}$ is infinite. Then $\pi_{a, a^{\prime}} \circ \pi(n)=\pi(n)$ and $\pi_{a, a^{\prime}}(n) \neq n$ for any $n \in \pi^{-1}\left(X \backslash a^{\prime}\right)$. From the last inequality it follows that $\pi\left(\pi_{a, a^{\prime}}(n)\right) \neq \pi(n)$ and hence $\left[\pi_{a, a^{\prime}}\right]_{\mathbb{F}}$ does not commute with $[\pi]_{\mathbb{F}}$.

Claim 3. If $a \in \mathcal{A}$ is such that $\operatorname{supp}(\pi) \cap a$ is infinite then there is some $i \in \mathbb{Z}$ such that $\pi\left\lceil a \equiv{ }^{*} \pi_{a}^{i}\lceil a\right.$.

Proof. From Claim 1 and Claim 2 it follows that for almost all $n \in a$ there is some $k(n)$ such that $\pi(n)=\pi_{a}^{k(n)}(n)$. If the claim is false then there are infinitely many $n \in a$ such that $k(n) \neq k\left(\pi_{a}(n)\right)$. For any such $n$ it follows that

$$
\pi_{a} \circ \pi(n)=\pi \circ \pi_{a}^{k(n)}(n)=\pi_{a}^{k(n)+1}(n) \neq \pi_{a}^{k\left(\pi_{a}(n)\right)+1}(n)=\pi_{a}^{k\left(\pi_{a}(n)\right)}\left(\pi_{a}(n)\right)=\pi \circ \pi_{a}(n)
$$

and hence $\pi_{a} \circ \pi$ and $\pi \circ \pi_{a}$ disagree on infinitely many integers.

There are now two cases to consider.

Case One. There is a finite subset $\left\{a_{1}, a_{2}, \ldots a_{n}\right\} \subseteq \mathcal{A}$ such that $\operatorname{supp}(\pi) \subseteq^{*} \bigcup_{i=1}^{n} a_{i}$.

In this case, use Claim 3 to choose integers $k_{i} \in \mathbb{Z}$ such that

$$
\pi \uparrow a_{i} \equiv^{*} \pi_{a_{i}}^{k_{i}}
$$

for each $i \leq n$. This contradicts that $[\pi]_{\mathbb{F}} \notin \Phi(F(\mathcal{A}))$.

Case Two. There is no finite subset $\left\{a_{1}, a_{2}, \ldots a_{n}\right\} \subseteq \mathcal{A}$ such that $\operatorname{supp}(\pi) \subseteq^{*} \bigcup_{i=1}^{n} a_{i}$. 
In this case there are uncountably many $a \in \mathcal{A}$ such that $\operatorname{supp}(\pi) \cap a$ is infinite. Use Claim 3 to conclude that there is some $i \in \mathbb{Z}$ such that, without loss of generality, $\pi\left\lceil a \equiv^{*} \pi_{a}^{i}\right.$ for uncountably many $a \in \mathcal{A}$. Hence there is some $k \in \mathbb{N}$ such that $\pi\left\lceil\{n \in a: n \geq k\}=\pi_{a}^{i} \uparrow\{n \in a: n \geq k\}\right.$ for uncountably many $a \in \mathcal{A}$. Hence there are distinct $a$ and $b$ in $\mathcal{A}$ such that it is possible to choose $j \in\{n \in a: n \geq k\} \cap\{n \in b: n \geq k\}$ such that $\pi(j)=\pi_{a}^{i}(j) \neq \pi_{b}^{i}(j)=\pi(j)$.

\section{A LOWER BOUND}

The next series or preliminary lemmas will be used in the proof of Theorem 3.2 which establishes a lower bound for $A\left([\mathbb{N}]^{<\aleph_{0}}\right)$. Corollary 3.1 has as a trivial consequence the fact that any maximal Abelian subgroup of the full symmetric group of the integers has cardinality $2^{\aleph_{0}}$; however, this can also be shown by using the topology of pointwise convergence on this group and noting that any maximal Abelian subgroup must be closed, and hence have cardinality $2^{\aleph_{0}}$.

Lemma 3.1. Let $\mathcal{S}$ be a finite subset of $\mathbb{S}$ whose elements almost commute with each other.

(1) If all the orbits of each $\pi \in \mathcal{S}$ are finite then each element of $\Omega_{\mathcal{S}}$ is finite.

(2) If, in addition, for each $\pi \in \mathcal{S}$ all the orbits of $\pi$ have size less than or equal to $m(\pi)$ then the cardinality of all but finitely many elements of $\Omega_{\mathcal{S}}$ is no greater than $\prod_{\pi \in \mathcal{S}} m(\pi)$.

Proof. Proceed by induction on $n=|\mathcal{S}|$, the case $n=1$ being trivial. If the lemma is true for $n$ let $\mathcal{S}=\left\{\pi_{i}\right\}_{i=1}^{n+1}$ and let $\mathcal{S}^{\prime}=\left\{\pi_{i}\right\}_{i=1}^{n}$. Define

$$
B=\bigcup_{i=1}^{n} \bigcup_{j=i}^{n+1} \mathrm{NC}\left(\pi_{i}, \pi_{j}\right)
$$

and, if the orbits of each $\pi \in \mathcal{S}$ are bounded by $m(\pi)$ then let $B^{\prime}$ be the union of those finitely many $A \in \Omega_{\mathcal{S}^{\prime}}$ whose cardinality is not bounded by $\prod_{i=1}^{n} m\left(\pi_{i}\right)$. Define

$$
B^{*}=\Omega_{\mathcal{S}^{\prime}}\left(\operatorname{orb}_{\pi_{n+1}}\left(B \cup B^{\prime}\right)\right) .
$$

Observe that $B^{*}$ is finite by the induction hypothesis and the fact the orbits of $\pi_{n+1}$ are finite. Hence, it suffices to show that if $C \in \Omega_{\mathcal{S}^{\prime}}$ and $C \cap B^{*}=\emptyset$ then $C^{\prime}=\operatorname{orb}_{\pi_{n+1}}(C)$ belongs to $\Omega_{\mathcal{S}}$. The fact that it is finite is immediate from the hypothesis that all orbits of $\pi_{n+1}$ are finite; similarly, if $|C| \leq \prod_{i=1}^{n} m\left(\pi_{i}\right)$ then it follows that $\left|C^{\prime}\right| \leq \prod_{i=1}^{n+1} m\left(\pi_{i}\right)$.

To see that $C^{\prime} \in \Omega_{\mathcal{S}}$ it suffices to show that if $i \leq n$ and $c \in C^{\prime}$ then $\operatorname{orb}_{\pi_{i}}(c) \subseteq C^{\prime}$. If not then there is some $d \in C$ such that $c \in \operatorname{orb}_{\pi_{n+1}}(d)$. Since $\operatorname{orb}_{\pi_{i}}(d) \subseteq C \subseteq C^{\prime}$ it follows that there must be some $e \in \operatorname{orb}_{\pi_{n+1}}(d)$ such that $\pi_{i}(e) \in C^{\prime}$ and $\pi_{i}\left(\pi_{n+1}(e)\right) \notin C^{\prime}$. But $\pi_{n+1}\left(\pi_{i}(e)\right) \in C^{\prime}$ by definition. Hence $\pi_{n+1} \circ \pi_{i}(e) \neq \pi_{i} \circ \pi_{n+1}(e)$ contradicting that $e \notin B$.

Lemma 3.2. Let $\mathcal{S} \subseteq \mathbb{S}$ be finite and suppose that $\pi \in \mathbb{S}$ and $\theta \in \mathbb{S}$ almost commute with each member of $S$. Then there is a finite set $Y$ such that if $\pi \uparrow X \cup Y=\theta \uparrow X \cup Y$ then $\pi \uparrow \Omega_{\mathcal{S}}(X)=\theta \uparrow \Omega_{\mathcal{S}}(X)$. Moreover, if $\pi$ and $\theta$ actually commute with each member of $S$ then $Y$ can be taken to be the empty set.

Proof. Let $Y^{\prime}=\bigcup_{\sigma \in \mathcal{S}} \mathrm{NC}(\sigma, \pi) \cup \mathrm{NC}(\sigma, \theta)$ and let $Y=\bigcup_{\sigma \in \mathcal{S}} \sigma\left(Y^{\prime}\right) \cup \sigma^{-1}\left(Y^{\prime}\right)$. Note that $\Omega_{\mathcal{S}}(X \cup Y)=$ $\bigcup_{i=0}^{\infty} X^{(i)}$ where $X^{(0)}=X \cup Y$ and $X^{(n+1)}=\bigcup_{\sigma \in \mathcal{S}} \operatorname{orb}_{\sigma}\left(X^{(n)}\right)$ and, hence, it suffices to show by induction that $\pi \uparrow X^{(n)}=\theta \uparrow X^{(n)}$ for each $n$ assuming that $\pi \uparrow X^{(0)}=\theta \uparrow X^{(0)}$. To this end, suppose that $\pi \uparrow X^{(n)}=\theta \uparrow X^{(n)}$ and $x \in X^{(n+1)}$. Then there is some $\bar{x} \in X^{(n)}$ and $\sigma \in \mathcal{S}$ such that $x \in \operatorname{orb}_{\sigma}(\bar{x})$. But $\pi(\bar{x})=\theta(\bar{x})$ and hence $\sigma^{k}(\pi(\bar{x}))=\sigma^{k}(\theta(\bar{x}))$ for any $k$. If $n>1$ then $\bar{x} \notin Y$ and it follows that $\pi\left(\sigma^{k}(\bar{x})\right)=\theta\left(\sigma^{k}(\bar{x})\right)$ for all $k$. Since $x=\sigma^{k}(\bar{x})$ for some $k$ the result follows.

If $n=1$ it will be shown by induction on $|k|$ that if $x \in X^{(1)}$ and $\bar{x} \in X^{(0)}$ and $\sigma \in \mathcal{S}$ are such that $x=\sigma^{k}(\bar{x})$ then $\theta(x)=\pi(x)$. If $|k|=0$ this is immediate. First assume that $k>0$ and $\theta\left(\sigma^{k-1}(\bar{x})\right)=\pi\left(\sigma^{k-1}(\bar{x})\right)$. If $\sigma^{k-1}(\bar{x}) \notin Y^{\prime}$ then $\sigma^{k-1}(\bar{x}) \notin \mathrm{NC}(\pi, \sigma) \cup \mathrm{NC}(\theta, \sigma)$ and so

$$
\theta\left(\sigma^{k}(\bar{x})\right)=\sigma\left(\theta\left(\sigma^{k-1}(\bar{x})\right)\right)=\sigma\left(\pi\left(\sigma^{k-1}(\bar{x})\right)\right)=\pi\left(\sigma^{k}(\bar{x})\right)
$$

as required. On the other hand, if $\sigma^{k-1}(\bar{x}) \in Y^{\prime}$ then $\sigma\left(\sigma^{k-1}(\bar{x})\right) \in Y$ and so $\theta\left(\sigma^{k}(\bar{x})\right)=\pi\left(\sigma^{k}(\bar{x})\right)$ in this case also. The case that $k<0$ is handled similarly. 
Definition 3.1. If $H \subseteq \mathbb{S}$ is a subgroup then define $H$ to be strongly almost Abelian if and only if for each $h \in H$ there is a finite set $F(h) \subseteq \mathbb{N}$ such that if and $h_{1}$ and $h_{2}$ belong to $H$ then $\mathrm{NC}\left(h_{1}, h_{2}\right) \subseteq F\left(h_{1}\right) \cup F\left(h_{2}\right)$.

Lemma 3.3. If $H \subseteq \mathbb{S}$ is an uncountable subgroup and $F: H \rightarrow[\mathbb{N}]^{<\aleph_{0}}$ attests to the fact that $H$ is strongly almost Abelian then there is a perfect set $P \subseteq \mathbb{S}$ and a finite $W \subseteq \mathbb{N}$ such that:

- There is some $g^{*} \in H$ such that for all $n \in \mathbb{N} \backslash W$ and $\pi \in P$ either $\pi(n)=n$ or $\pi(n)=g^{*}(n)$.

- $N C(\pi, h) \subseteq W \cup F(h) \cup h^{-1}(W)$ for $\pi \in P$ and $h \in H$.

Moreover if $H$ is actually Abelian and not just strongly almost Abelian then $W$ can be assumed to be empty and it can be concluded that each $\pi \in P$ commutes with each $h \in H$.

Proof. Given $X \subseteq \mathbb{N}$ and a finite $W \subseteq \mathbb{N}$ define $c l_{W}^{0}(X)=X$,

$$
c l_{W}^{1}(X)=\left\{z \in \mathbb{N} \backslash W:(\exists h \in H)(\exists x \in X \backslash F(h)) z=h(x) \text { and } z \notin F\left(h^{-1}\right)\right\}
$$

and let $c l_{W}^{n+1}(X)=c l_{W}^{1}\left(c l_{W}^{n}(X)\right)$ and then, let $c l_{W}(X)=\bigcup_{i=1}^{\infty} c l_{W}^{i}(X)$. Observe first that it follows from an argument similar to that in Lemma 3.2 that, if $F\left(g_{1}\right) \subseteq W$ and $F\left(g_{2}\right) \subseteq W$ and $g_{1} \uparrow X=$ $g_{2} \uparrow X$ then $g_{1}\left\lceil c l_{W}^{1}(X)=g_{2}\left\lceil c l_{W}^{1}(X)\right.\right.$ and hence, $g_{1} \uparrow c l_{W}(X)=g_{2} \uparrow c l_{W}(X)$.

If, for every $W \in[\mathbb{N}]^{<\aleph_{0}}$ there is some $A_{W} \in[\mathbb{N}]^{<\aleph_{0}}$ such that $A_{W} \cup c l_{W}\left(A_{W}\right)=\mathbb{N}$ then it follows that each $g \in H$ is determined by its values on $F(g) \cup A_{F(g)}$. This contradicts that $H$ is uncountable.

Therefore it must be the case that there is some $W \in[\mathbb{N}]^{<\aleph_{0}}$ such that $c l_{W}(A) \neq \mathbb{N}$ for every $A \in[\mathbb{N}]^{<\aleph_{0}}$. Hence, it is possible to choose some $W^{\prime} \supseteq W$ such that the set of all $g \in H$ such that $F(g) \subseteq W^{\prime}$ is uncountable. Observe that $c l_{W}(A) \supseteq \operatorname{cl}_{W^{\prime}}(A)$ for any $A$, so it is possible to select $\left\{a_{i}\right\}_{i=1}^{\infty} \subseteq \mathbb{N}$ be such that $\left\{c l_{W^{\prime}}\left(\left\{a_{i}\right\}\right)\right\}_{i=1}^{\infty}$ is an infinite family. Observe that if $g \in H$ is such that $F(g) \subseteq W^{\prime}$ and $g\left\lceil c l_{W^{\prime}}\left(\left\{a_{i}\right\}\right)\right.$ is the identity for all but finitely many $i$ then $g$ is determined by its values on $F(g) \cup\left\{a_{i}:\left(\exists n \in c l_{W^{\prime}}\left(\left\{a_{i}\right\}\right)\right) g(n) \neq n\right\}$. Hence, there must be some $\bar{g} \in H$ such that $F(\bar{g}) \subseteq W^{\prime}$ and $\bar{g}\left\lceil c l_{W^{\prime}}\left(\left\{a_{i}\right\}\right)\right.$ is not the identity for infinitely many $i$. Let

$$
Z=\left\{i \in \mathbb{N}:\left(\exists n \in c l_{W^{\prime}}\left(\left\{a_{i}\right\}\right)\right) \bar{g}(n) \neq n \text { and } \bar{g}^{-1}\left(W^{\prime}\right) \cap c l_{W^{\prime}}\left(\left\{a_{i}\right\}\right)=\emptyset\right\} .
$$

First notice that it follows from the definition of $c l_{W^{\prime}}$ and the inclusion $F(\bar{g}) \subseteq W^{\prime}$ that $\bar{g}\left(c l_{W^{\prime}}\left(\left\{a_{i}\right\}\right)\right) \subseteq$ $c l_{W^{\prime}}\left(\left\{a_{i}\right\}\right) \cup W^{\prime}$. Hence $\bar{g} \uparrow c l_{W^{\prime}}\left(\left\{a_{i}\right\}\right)$ is a permutation of $c l_{W^{\prime}}\left(\left\{a_{i}\right\}\right)$ for each $i \in Z$. Therefore, if for each $t: Z \rightarrow 2$ the function $g_{t}$ is defined

$$
g_{t}(n)= \begin{cases}\bar{g}(n) & \text { if } n \in c l_{H, W^{\prime}}\left(\left\{a_{i}\right\}\right) \text { and } t(i)=0 \\ n & \text { otherwise. }\end{cases}
$$

then $g_{t}$ is a permutation of $\mathbb{N}$. It is routine to check that each $g_{t}(h(n))=h\left(g_{t}(n)\right)$ provided that $n \notin W^{\prime} \cup F(h) \cup h^{-1}\left(W^{\prime}\right)$.

Corollary 3.1. If $H \subseteq \mathbb{S}$ is an uncountable, maximal strongly almost Abelian subgroup then $|H|=$ $2^{\aleph_{0}}$.

Proof. The maximality of $H$ implies that it must contain the perfect set of the conclusion of Lemma 3.3 .

Lemma 3.4. If $H$ is a maximal Abelian subgroup of $\mathbb{S} / \mathbb{F}$ and there are

$$
\left\{\left[\pi_{1}\right]_{\mathbb{F}},\left[\pi_{2}\right]_{\mathbb{F}}, \ldots\left[\pi_{n}\right]_{\mathbb{F}}\right\} \subseteq H
$$

such that, letting $\mathcal{S}=\left\{\pi_{1}, \pi_{2}, \ldots \pi_{n}\right\}$, there are infinitely many different cardinalities of equivalence classes of $\equiv \mathcal{S}$, then $|H|=2^{\aleph_{0}}$.

Proof. Let $A_{j}=\cup\left(\Omega_{\mathcal{S}} \cap[\mathbb{N}]^{j}\right)$ and note that $\left\{A_{j}\right\}_{j=0}^{\infty}$ is an infinite set. For each $j \geq 2$ choose some $i \leq n$ such that $\pi_{i}\left\lceil A_{j}\right.$ is different from the identity on an infinite subset of $A_{j}$. For each $F: \mathbb{N} \rightarrow 2$ define

$$
\theta_{F}(k)= \begin{cases}\pi_{j}(k) & \text { if } k \in A_{j} \text { and } F(j)=1 \\ n & \text { otherwise }\end{cases}
$$

and observe that $\theta_{F}$ is a bijection. It suffices to show that $\mathrm{NC}\left(\theta_{F}, \pi\right)$ is finite for each $\pi \in H$. 
To see that this is so, let $\pi \in H$ and let $j$ be so large that

$$
\left(\bigcup_{i=1}^{n} \mathrm{NC}\left(\pi_{i}, \pi\right)\right) \cap\left(\bigcup_{i=1}^{\infty} A_{i}\right) \subseteq \bigcup_{i=1}^{j} A_{i} .
$$

Hence, if $k \geq j$ then $\pi \uparrow A_{k}$ commutes with $\pi_{i} \uparrow A_{k}$ for $i \leq n$. From this it follows that $\pi \uparrow A_{k}$ is a permutation of $A_{k}$ because, if $\pi(a) \notin A_{k}$ then $\pi$ is an $\mathcal{S}$-isomorphism from $\Omega_{\mathcal{S}}(a)$ onto the $\Omega_{\mathcal{S}}(\pi(a))$. This contradicts that $\left|\Omega_{\mathcal{S}}(a)\right|=k \neq\left|\Omega_{\mathcal{S}}(\pi(a))\right|$.

Definition 3.2. If $g \in \mathbb{S}$ then define $I(g)=\bigcup\left\{\operatorname{orb}_{g}(n):\left|\operatorname{orb}_{g}(n)\right|=\aleph_{0}\right\}$. For a finite set $\mathcal{S} \subseteq \mathbb{S}$ define $I^{*}(\mathcal{S})=\Omega_{\mathcal{S}}\left(\bigcup_{\sigma \in \mathcal{S}} I(\sigma)\right)$.

Lemma 3.5. If $H \subseteq \mathbb{S}$ is an uncountable, maximal, almost commuting subgroup of size less than $2^{\aleph_{0}}$ then $[\mathbb{N}]^{<\aleph_{0}} \cup\left\{I^{*}(\mathcal{S})\right\}_{\mathcal{S} \in[H]<\aleph_{0}}$ generates a proper ideal.

Proof. If not, let $B \subseteq H$ and $C \subseteq \mathbb{N}$ be finite sets such that $I^{*}(B) \cup C=\mathbb{N}$. Without loss of generality it may be assumed that $\mathrm{NC}\left(b, b^{\prime}\right) \subseteq C$ for each $b$ and $b^{\prime}$ in $B$. Let $S=\left\{A \in \Omega_{B}: A \cap C=\emptyset\right\}$. Observe that each set in $S$ is infinite since it must intersect some $I(b)$ where $b \in B$. Moreover, $S$ itself is an infinite set since Lemma 3.2 would imply that $H$ is countable otherwise.

Now let $\mathbb{S}_{S}$ be the symmetric group on $S$ and define $\Phi: H \rightarrow \mathbb{S}_{S}$ by $\Phi(h)(s)=t$ if and only if $h(s) \equiv^{*} t$. Observe that $\Phi$ is well defined. To see this suppose that $s \in S$ and $h \in H$ and there are distinct $t$ and $t^{\prime}$ in $S$ such that $|h(s) \cap t|=|h(s) \cap t|=\aleph_{0}$. Then there exist $i$ and $j$ in $s$ such that $h(b(i))=b(h(i))$ for all $b \in B$ and $h(i) \in t$ and $h(j) \in t^{\prime}$. But then, since $\{i, j\} \subseteq s \in \Omega_{B}$, there is some $g$ in the subgroup generated by $B$ such that $g(i)=j$. Hence $h(j)=h(g(i))=g(h(i))$. Furthermore, $h(i) \in t$ and $g$ in the subgroup generated by $B$ together imply that $g(h(i)) \in t$. However, $h(j) \in t^{\prime}$ so $h(g(i)) \neq g(h(i))$ contradicting the choice of $i$. A similar argument shows that $\Phi$ is a homomorphism.

Moreover, its image $\Phi(H)$ is an Abelian subgroup of $\mathbb{S}_{S}$. To see this, let $s \in S$. If $\Phi(g)(\Phi(h)(s)) \neq$ $\Phi(h)(\Phi(g)(s))$ then, $g(h(s)) \not^{*} h(g(s))$ and hence there are infinitely many $i \in s$ such that $g(h(i)) \neq$ $h(g(i))$ contradicting that $h$ almost commutes with $g$.

To begin it will be shown that there cannot be a perfect set $P \subseteq \mathbb{S}_{S}$ such that:

(1) There is some $g^{*} \in H$ such that for all $s \in S$ and $\pi \in P$ either $\pi(s)=s$ or $\pi(s)=\Phi\left(g^{*}\right)(s)$.

(2) Every element of $P$ commutes with every element of $h \in H$.

To see this suppose that $P$ and $g^{*}$ contradict the assertion. For $\pi \in P$ define $\pi^{*} \in \mathbb{S}$ by

$$
\pi^{*}(i)= \begin{cases}i & \text { if } i \in s \in S \text { and } \pi(s)=s \\ g^{*}(i) & \text { if } i \in s \in S \text { and } \pi(s) \neq s\end{cases}
$$

It suffices to show that $\pi^{*}$ almost commutes with each $h \in H$. To see that this is so let $i \in \mathbb{N} \backslash \mathrm{NC}\left(g^{*}, h\right)$ and let $s \in S$ be such that $i \in s$. If $\pi(s)=s$ then $h\left(\pi^{*}(i)\right)=h(i)=\pi(h(i))$ the last equality holding because $h(i) \in \Phi(h)(s)$ and $\pi(\Phi(h)(s))=\Phi(h)(\pi(s))=\Phi(h)(s)$. On the other hand, if $\pi(s) \neq s$ then $h\left(\pi^{*}(i)\right)=h\left(g^{*}(i)\right)=g^{*}(h(i))=\pi^{*}(h(i))$ the last equality holding because $h(i) \in \Phi(h)(s)$ and $\pi(\Phi(h)(s))=\Phi(h)(\pi(s)) \neq \Phi(h)(s)$.

To see that $\Phi(H)$ is not countable suppose otherwise. To begin, notice that there must be some $A \in \Omega_{\Phi(H)}$ such that $\{h \uparrow(\cup A)\}_{h \in H}$ is uncountable - keep in mind that $A \subseteq \Omega_{B}$. This so because if not, then it is easy to find $P$ and $g^{*}$ satisfying conditions 1 and 2 . Simply let $g^{*} \in H$ be any permutation $\Phi\left(g^{*}\right) \uparrow A$ which is different from the identity on infinitely many sets in $\Omega_{\Phi(H)}$. Then let $P$ be the set if all $g \in \mathbb{S}_{S}$ such that for all $A \in \Omega_{\Phi(H)}$ either $g\left\lceil A=\Phi\left(g^{*}\right) \uparrow A\right.$ or else $g\lceil A$ is the identity. If no such $g^{*}$ exists then it follows that $H$ is countable because $\{h \uparrow(\cup A)\}_{h \in H}$ is countable for each $A \in \Omega_{\Phi(H)}$ and each $h$ is the identity on all but finitely many $A \in \Omega_{\Phi(H)}$.

Hence, there must be some $A \in \Omega_{\Phi(H)}$ such that $\{h \uparrow(\cup A)\}_{h \in H}$ is uncountable and hence there is some $h^{*} \in H$ such that $\left\{h \uparrow \cup A: \Phi(h) \uparrow A=\Phi\left(h^{*}\right) \uparrow A\right\}$ is uncountable. Observe that if $\Phi(h)\left\lceil A=\Phi\left(h^{\prime}\right) \uparrow A\right.$ and there is some $i \in \cup A$ such that $h(i)=h^{\prime}(i)$ then $h \uparrow \cup A \equiv{ }^{*} h^{\prime} \uparrow \cup A$. To see this note first that if $\{i, j\} \subseteq s \in A$ then there is some $b$ in the group generated by $B$ such that $b(i)=j$. Since $s \notin C$ it follows that $h(j)=h(b(i))=b(h(i))=b\left(h^{\prime}(i)\right)=h^{\prime}(j)$. If $j \in \cup A$ then there are $s_{i}$ and $s_{j}$ in $A$ such that $i \in s_{i}$ and $j \in s_{j}$ and there is $\bar{h} \in H$ such that $\Phi(\bar{h})\left(s_{i}\right)=s_{j}$. Since $s_{i}$ is infinite, there is some $i^{*} \in s_{i} \backslash\left(\mathrm{NC}(\bar{h}, h) \cup \mathrm{NC}\left(\bar{h}, h^{\prime}\right)\right)$ such that $\bar{h}\left(i^{*}\right) \in s_{j}$. Hence 
$h\left(\bar{h}\left(i^{*}\right)\right)=\bar{h}\left(h\left(i^{*}\right)\right)=\bar{h}\left(h^{\prime}\left(i^{*}\right)\right)=h^{\prime}\left(\bar{h}\left(i^{*}\right)\right)$ and, since $\left\{\bar{h}\left(i^{*}\right), j\right\} \subseteq s_{j}$ it follows that $h(j)=h^{\prime}(j)$. But, since $\left\{h\left\lceil(\cup A): \Phi(h)\left\lceil A=\Phi\left(h^{*}\right)\lceil A\}\right.\right.\right.$ is uncountable it is possible to find $h$ and $h^{\prime}$ such that there are $i$ and $j$ in $\cup A$ such that $h(i)=h^{\prime}(i), h(j) \neq h^{\prime}(j)$ and $\Phi(h)\left\lceil A=\Phi\left(h^{*}\right)\lceil A\right.$.

Since $\Phi(H)$ is uncountable and Abelian it follows from Lemma 3.3 that there exist $P$ and $g^{*}$ satisfying the conditions 1 and 2 .

The following alternate characterization, due to M. Bell, of the cardinal invariant $\mathfrak{p}$ of Definition 1.2 will be used in the proof of Theorem 3.2 .

Theorem 3.1. The cardinal $\mathfrak{p}$ is the least cardinal such that there is a $\sigma$-centered partially ordered sef] $\mathbb{P}$ and a collection $D$ of $\mathfrak{p}$ dense subsets of $\mathbb{P}$ for which there is no centred subset $G \subseteq \mathbb{P}$ intersecting each member of $D$.

Proof. See [3].

Theorem 3.2. If $H \subseteq \mathbb{S} / \mathbb{F}$ is an uncountable, maximal Abelian subgroup then $|H| \geq \mathfrak{p}-$ in other words, $A\left([\mathbb{N}]^{<\aleph_{0}}\right) \geq \mathfrak{p}$.

Proof. If $H \subseteq \mathbb{S} / \mathbb{F}$ is an uncountable, maximal Abelian subgroup and $|H|<\mathfrak{p}$ then it follows from Lemma 3.5 that $\left\{I^{*}(\mathcal{S}):\{[\sigma]: \sigma \in \mathcal{S}\} \in[H]^{<\aleph_{0}}\right\}$ generates a proper ideal.

Let $\mathbb{P}$ be the partial order consist of all $p=\left(h^{p}, \mathcal{S}^{p}\right)$ such that:

(1) $h^{p}$ is a finite involution

(2) $\mathcal{S}^{p}$ is a finite subset such that if $\sigma \in \mathcal{S}^{p}$ then $[\sigma] \in H$

and define $p \leq q$ if and only if

(1) $h^{p} \supseteq h^{q}$

(2) $\mathcal{S}^{p} \supseteq \mathcal{S}^{q}$

(3) the domain of $h^{p} \backslash h^{q}$ is disjoint from $I^{*}\left(\mathcal{S}^{q}\right)$

(4) if $j$ is in the domain of $h^{p} \backslash h^{q}$ and $\sigma \in \mathcal{S}^{q}$ then $\sigma(j)$ is in the domain of $h^{p} \backslash h^{q}$ and $\sigma\left(h^{p}(j)\right)=h^{p}(\sigma(j)$.

It is clear that $\mathbb{P}$ is $\sigma$-centred. Moreover, the sets $D_{\pi}=\left\{p \in \mathbb{P}: \pi \in \mathcal{S}^{p}\right\}$ are all dense. Furthermore, so are the sets

$$
E_{n}=\left\{p \in \mathbb{P}: n \in \operatorname{domain}\left(h^{p}\right) \cup I^{*}\left(\mathcal{S}^{p}\right)\right\} .
$$

To see that this is so, let $p \in \mathbb{P}$ be given and suppose that $n \notin I^{*}\left(\mathcal{S}^{p}\right)$. This implies that the $\equiv_{I^{*}}\left(\mathcal{S}^{p}\right)^{-}$ equivalence class of $n$ is finite by Lemma 3.1. Now let $h^{q}$ be the union of $h^{p}$ and the identity on the

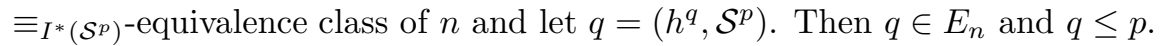

Hence, if $|H|<\mathfrak{p}$ then there is a filter $G \subseteq \mathbb{P}$ meeting each $D_{\pi}$ for $\pi \in H$ and $E_{n}$ for $n \in \mathbb{N}$. Define $\pi_{G}: \mathbb{N} \rightarrow \mathbb{N}$ by

$$
\pi_{G}(j)= \begin{cases}h^{p}(j) & \text { if } \quad(\exists p \in G) j \in \operatorname{domain}\left(h^{p}\right) \\ j & \text { if } \quad(\forall p \in G) j \notin \operatorname{domain}\left(h^{p}\right)\end{cases}
$$

It is easily verified that $\pi_{G} \in \mathbb{S}$. To see that $\pi_{G}$ almost commutes with each member of $H$ let $\pi \in H$. Let $p \in G$ be such that $\pi \in \mathcal{S}^{p}$. Then if $j \in \mathbb{N} \backslash$ domain $\left(h^{p}\right.$ there are two possibilities. If there is some $q \in G$ such that $j$ belongs to the domain of $h^{q}$ it is clear that $\pi\left(\pi_{G}(j)\right)=\pi\left(h^{q}(j)\right)=$ $h^{q}(\pi(j))=\pi_{G}(p i(j))$. However, if there is no $q \in G$ such that $j$ belongs to the domain of $h^{q}$ then, by virtue of the fact that $E_{j} \cap G \neq \emptyset$, it must be the case that there is some $q \in G$ such that $j \in I^{*}\left(\mathcal{S}^{q}\right)$. Since $\pi \in \mathcal{S}^{q}$ it follows that $\pi(j) \in \mathcal{S}^{q}$. Hence $\pi_{G}(j)=j$ and $\pi_{G}(\pi(j))=\pi(j)$ and so $\pi\left(\pi_{G}(j)\right)=\pi\left(h^{q}(j)\right)=h^{q}(\pi(j))=\pi_{G}(p i(j))$.

All that remains to be shown is that the following sets are dense

$$
D_{\pi, k}=\left\{p \in \mathbb{P}:(\exists j \geq k) h^{p}(j) \neq \pi(k)\right\}
$$

for $\pi \in H$ and $k \in \mathbb{N}$. To establish this, let $p \in \mathbb{P}$ be given. By Lemma 3.1 it follows that each $\equiv_{\mathcal{S}^{p}}$-equivalence class which is disjoint from $I^{*}\left(\mathcal{S}^{p}\right)$ is finite. Moreover, by Lemma 3.4 there must be

\footnotetext{
${ }^{3}$ A partially ordered set is said to be $\sigma$-centred if it is the union of countably many centred subsets - in other words, it is the union of countably many subsets which contain a lower bound for any two of their elements.

${ }^{4}$ In other words, $h^{p}$ is it own inverse.
} 
infinitely many of the same cardinality, and, hence there must be two $\equiv_{\mathcal{S}^{p} \text {-equivalence classes } A \text { and }}$ $B$ such that:

(1) both $A$ and $B$ are disjoint from $I^{*}\left(\mathcal{S}^{p}\right)$

(2) both $A$ and $B$ are disjoint from the domain of $h^{p}$

(3) $k<\min (A)$

(4) $k<\min (B)$

(5) $A$ and $B$ are $\mathcal{S}$-isomorphic.

There are two possibilities. If $\Phi=\pi \uparrow A$ then let let $h^{q}$ be the union of $h^{p}$ and the identity on $A$ and let $q=\left(h^{q}, \mathcal{S}^{p}\right)$. Otherwise, let $h^{q}=h^{p} \cup \Phi \cup \Phi^{-1}$ and let $q=\left(h^{q}, \mathcal{S}^{p}\right)$. In either case $q \leq p$ and $q \in D_{\pi, k}$.

\section{4. $A\left([\mathbb{N}]^{<\aleph_{0}}\right)$ CAN BE SMALleR THAN $\mathfrak{a}$}

Through this section the notation $f \leq^{*} g$ will be used to denote the relation of eventual domination — in other words, $f(n) \leq g(n)$ for all but finitely many $n$.

Definition 4.1. A partial order $\mathbb{P}$ will be said to be weakly dominated over the model $V$ if for every $H: \mathbb{N} \rightarrow \mathbb{N}$ belonging to $V^{\mathbb{P}}$ either there is $g: \mathbb{N} \rightarrow \mathbb{N}$ in $V$ such that $H \leq^{*} g$ or for every $f: \mathbb{N} \rightarrow \mathbb{N}$ belonging to $V$ there is some $R \in \prod_{n=1}^{\infty}[\mathbb{N}]^{\leq n}$ belonging to $V$ such that the following conditions hold:

- $f\left(\max \left(\bigcup_{i \in n} R(i)\right)\right)<\min (R(n))$

- there are infinitely many $n$ such that $H \cap(n \times R(n)) \neq \emptyset$.

Lemma 4.1. If $G \in \mathbb{L}_{\alpha}$ is generic over $V, f \in \omega_{\omega}$ belongs to $V[G]$ and there is $g \in \omega_{\omega}$ in $V$ such that $f \leq^{*} g$ then there is $f^{*} \in \omega_{\omega}^{\omega}$ in $V$ such that $\left|f \cap f^{*}\right|=\aleph_{0}$.

Proof. Let $\left\{n_{i}\right\}_{i=0}^{\infty}$ be an increasing sequence of integers such that $n_{i+1}-n_{i}<n_{i+2}-n_{i+1}$ for each $i$. In $V[G]$ let the function $\bar{f}$ be defined on $\mathbb{N}$ by $\bar{f}(i)=f \uparrow\left[n_{i}, n_{i+1}\right)$. As $f$ is bounded by $g \in V$ it follows from well known properties of Laver forcing that there is $\bar{f}^{*} \in V$ such that $\bar{f}^{*}(i) \in\left[\prod_{j=n_{i}}^{n_{i+1}-1} g(j)\right]^{i+1}$ and $\bar{f}(i) \in \bar{f}^{*}(i)$ for each $i \in \mathbb{N}$. It is then easy to define $f^{*}: \mathbb{N} \rightarrow \mathbb{N}$ in $V$ such that for all $i \in \mathbb{N}$ and $h \in \bar{f}^{*}(i)$ there is some $j \in\left[n_{i}, n_{i+1}\right)$ such that $f^{*}(j)=h(j)$. Hence, $f^{*}$ is the desired function.

Lemma 4.2. If $\mathbb{P}$ is weakly dominated over the model $V$ and $V^{\mathbb{P}}$ is a model of $2^{\aleph_{0}}=\aleph_{1}$ then there are permutations $\left\{p_{\xi}\right\}_{\xi \in \omega_{1}}$ of $\mathbb{N}$ in $V$ which mutually almost commute and which are maximal with respect to this property in $V^{\mathbb{P}}$.

Proof. Construct involutions $\left\{p_{\xi}\right\}_{\xi \in \omega_{1}} \subseteq \mathbb{S}$ by induction on $\xi$ such that any two almost commute.

Using the fact that $V^{\mathbb{P}}$ is a model of $2^{\aleph_{0}}=\aleph_{1}$ let $\left\{r_{\alpha}\right\}_{\alpha \in \omega_{1}}$ enumerate all $\mathbb{P}$-names for permutations of $\mathbb{N}$ which are forced not to belong to $V$ and suppose that $\left\{p_{\xi}\right\}_{\xi \in \eta}$ have been constructed.

Now let $\{\eta(i)\}_{i=1}^{\infty}$ enumerate $\eta$ and define

$$
\bar{\Omega}_{n}=\Omega_{\left\{p_{\eta(1)}, p_{\eta(2)}, \ldots, p_{\eta(n)}\right\}} \text { and } \bar{\Omega}_{n}(X)=\Omega_{\left\{p_{\eta(1)}, p_{\eta(2)}, \ldots, p_{\eta(n)}\right\}}(X)
$$

for any set $X$. Note that since the $p_{\xi}$ are almost commuting involutions it follows that $\Omega_{n} \subseteq^{*}[\mathbb{N}] \leq 2^{n}$. Now, for any $z \in[\mathbb{N}]^{<\aleph_{0}}$ let $\bar{\tau}_{n}(z)$ be the structure

$$
\left(\left|\bar{\Omega}_{n}(z)\right|,\left\{\Delta_{\bar{\Omega}_{n}(z)} \circ p_{\eta(i)} \circ \Delta_{\bar{\Omega}_{n}(z)}^{-1}\right\}_{i=1}^{n}\right)
$$

Since $\left\{\tau_{n}(\{j\})\right\}_{j \in \mathbb{N}}$ is finite for each $n$, it follows that, for all $j$, it is possible to choose a finite set $C(j) \subseteq \Omega_{j}$ such that

$$
\left\{\tau_{j}(\{i\}): i \in C(j)\right\}=\left\{\tau_{j}(\{i\}): i \in \mathbb{N} \text { and } \Omega_{j}(\{i\}) \cap j=\emptyset\right\}
$$

(4.3) $(\forall i \in \mathbb{N})\left(\forall i^{\prime} \in C(j)\right)$ if $\Omega_{j}(\{i\}) \cap j=\emptyset$ and $\tau_{j}(i)=\tau_{j}\left(i^{\prime}\right)$ then $\min \left(\Omega_{j}\left(\left\{i^{\prime}\right\}\right)<\min \left(\Omega_{j}(\{i\})\right)\right.$.

Now define a function $H$ in $V^{\mathbb{P}}$ by letting $H(j)$ be the least integer such that $H(j) \geq j$ and

(4.4) $(\exists i \in C(j)) \tau_{j}(\{i\})=\tau_{j}(\{H(j)\})$ and $\Delta_{\bar{\Omega}_{j}(\{i\})} \circ r_{\eta} \circ \Delta_{\bar{\Omega}_{j}(\{i\})}^{-1} \neq \Delta_{\bar{\Omega}_{j}(\{H(j)\})} \circ r_{\eta} \circ \Delta_{\bar{\Omega}_{j}(\{H(j)\})}^{-1}$. 
If no such integer exists then $r_{\eta}$ is completely determined by $r_{\eta}\left\lceil\bar{\Omega}_{j}(j \cup C(j))\right.$ and hence belongs to $V$ contradicting the fact that only names forced not to belong to $V$ were enumerated.

In $V$ define $f: \mathbb{N} \rightarrow \mathbb{N}$ as follows. First define $L(k)=\max \left(\bar{\Omega}_{k}(j)\right)$. Then let $f^{*}(k)$ be large enough that for each $z \in[\mathbb{N}]^{\leq k}$ there is $\bar{z} \in[\mathbb{N}]^{\leq k}$ such that

$$
\begin{aligned}
& \qquad\left|\bar{\Omega}_{k}(z)\right|=\left|\bar{\Omega}_{k}(\bar{z})\right| \\
& (4.6) \quad(\forall i \leq k)(\forall m \in z)(\exists \bar{m} \in \bar{z}) \Delta_{\bar{\Omega}_{k}(z), \bar{\Omega}_{k}(\bar{z})} \mid \bar{\Omega}_{i}(m) \text { is a bijection onto } \bar{\Omega}_{i}(\bar{m}) \\
& (4.7) \quad \bar{z} \subseteq\left[L(k), f^{*}(k)\right) \\
& \text { (4.8) } \quad(\forall \bar{m} \in \bar{z})(\exists m \in z) \Delta_{\bar{\Omega}_{k}(\bar{z}), \bar{\Omega}_{k}(z)}\left\lceil\bar{\Omega}_{i}(\bar{m}) \text { is a bijection onto } \bar{\Omega}_{i}(m)\right. \\
& \text { Finally, let } f(k)=L\left(f^{*}(k)\right)=\max \left(\bar{\Omega}_{k}\left(f^{*}(k)\right)\right) .
\end{aligned}
$$

There are two cases to consider. First assume that $H \leq{ }^{*} g$ for some $g \in{ }^{\omega} \omega \cap V$. In this case define $a_{n}$ inductively by setting $a_{0}=0$ and letting $a_{n+1}=L\left(f\left(a_{n}\right)\right)$. Using Lemma 4.1, find $H^{*} \in V$ such that $H\left(a_{n}\right)=H^{*}(n)$ for infinitely many integers $n$. By modifying $H^{*}$ if necessary, it may be assumed that $H^{*}(n) \leq f\left(a_{n}\right)$ for all $n$. Hence, $\bar{\Omega}_{k}\left(\left\{H^{*}(n)\right\}\right) \subseteq L\left(f\left(a_{n}\right)\right)=a_{n+1}$ for each $n$ and so, by the definition of $H, \bar{\Omega}_{k}\left(\left\{H^{*}(n)\right\}\right) \subseteq\left[a_{n}, a_{n+1}\right)$. Note that if $j$ is the unique element of $C\left(a_{n}\right)$ such that

$$
\tau_{a_{n}}(\{j\})=\tau_{a_{n}}\left(\left\{H^{*}(n)\right\}\right)
$$

then $a_{n} \leq j<a_{n+1}$ as well. Now construct the permutations $p_{\eta}^{n}$ of the interval $\left[a_{n}, a_{n+1}\right)$ so that

$$
\begin{gathered}
p_{\eta}^{n}\left\lceil\bar{\Omega}_{a_{n}}\left(\left\{H^{*}(n)\right\}\right)=\Delta_{\bar{\Omega}_{a_{n}}\left(\left\{H^{*}(n)\right\}\right), \bar{\Omega}_{a_{n}}(\{j\})}\right. \\
p_{\eta}^{n}\left\lceil\bar{\Omega}_{a_{n}}(\{j\})=\Delta_{\bar{\Omega}_{a_{n}}(\{j\}), \bar{\Omega}_{a_{n}}\left(\left\{H^{*}(n)\right\}\right)}\right.
\end{gathered}
$$

and $p_{\eta}^{n}$ is the identity elsewhere on $\left.\left[a_{n}, a_{n+1}\right)\right)$. Finally, let $p_{\eta}=\bigcup_{i=0}^{\infty} p_{\eta}^{i}$ and note that this is a well defined permutation. It is immediate that the composition $p_{\eta}^{i} \circ p_{\eta}^{i}$ is the identity and that for each $j \leq i, p_{\eta}^{i} \circ p_{\eta(j)}=p_{\eta(j)} \circ p_{\eta}^{i}$. Hence $p_{\eta}$ almost commutes with each $p_{\eta(j)}$. For any $n$ such that $H^{*}(n)=H\left(a_{n}\right)$ it follows that $p_{\eta}^{n}$ does not commute with $r_{\eta}\left\lceil\bar{\Omega}_{a_{n}}\left(\left\{H^{*}(n)\right\}\right)\right.$.

In the second case, suppose that $R \in \prod_{n=1}^{\infty}[\mathbb{N}]^{\leq n}$ is in $V$ and witnesses the conditions of Definition 4.1 with respect to $H$ and $f$. Let $\bar{R}(i)=\max \left(\bigcup_{j \in i} R(i)\right)$. The permutation $p_{\eta}$ will be defined so that $p_{\eta}=\bigcup_{i=0}^{\infty} p_{\eta}^{i}$ where the $p_{\eta}^{i}$ are constructed by induction so that

(1) The domain and range of $p_{\eta}^{i}$ are $[L(\bar{R}(i)), L(\bar{R}(i+1)))$.

(2) The composition $p_{\eta}^{i} \circ p_{\eta}^{i}$ is the identity.

(3) For each $j \leq i, p_{\eta}^{i} \circ p_{\eta(j)}=p_{\eta(j)} \circ p_{\eta}^{i}$.

To see that this can be done suppose that $\left\{p_{\eta}^{j}\right\}_{j \in i}$ have been constructed. Then the domain and range of $\bigcup_{j \in i} p_{\eta}^{j}$ are $L(\bar{R}(i))$ by the first induction hypothesis. Hence, $p_{\eta}^{i}$ will be defined so that its domain and range are $[L(\bar{R}(i)), L(\bar{R}(i+1))$. To see that this can be done recall that $R(i) \in[\mathbb{N}] \leq i$ and, furthermore, if $b \in R(i)$ then $b \geq f(\bar{R}(i))$ and so $\bar{\Omega}_{i}(\{b\}) \cap f^{*}(\bar{R}(i))=\emptyset$. The definition of $f^{*}$ guarantees that there is some $\bar{z} \in\left[\left[L(\bar{R}(i)), f^{*}(\bar{R}(i))\right)\right] \leq i$ such that $\left|\bar{\Omega}_{i}(\bar{z})\right|=\left|\bar{\Omega}_{i}(R(i))\right|$ and conditions 4.6 and 4.7 hold. Observe that $\bar{\Omega}_{i}(\bar{z}) \cap \bar{\Omega}_{i}(R(i))=\emptyset$ because $\bar{\Omega}_{i}(R(i)) \cap f^{*}(\bar{R}(i))=\emptyset$ and $\bar{\Omega}_{i}(\bar{z}) \subseteq f^{*}(\bar{R}(i))$. It follows that if $p_{\eta}^{i}$ is defined so that

$$
\begin{gathered}
p_{\eta}^{n}\left\lceil\bar{\Omega}_{i}(R(i))=\Delta_{\bar{\Omega}_{i}(R(i)), \bar{\Omega}_{i}(\bar{z})}\right. \\
p_{\eta}^{n}\left\lceil\bar{\Omega}_{i}(\bar{z})=\Delta_{\bar{\Omega}_{i}(\bar{z}), \bar{\Omega}_{i}(R(i))}\right.
\end{gathered}
$$

and $p_{\eta}^{n}$ is the identity elsewhere on $[L(\bar{R}(i)), L(\bar{R}(i+1)))$ then $p_{\eta}^{i}$ is a bijection of $[L(\bar{R}(i)), L(\bar{R}(i+$ 1))) and $p_{\eta}^{i} \circ p_{\eta}^{i}$ is the identity. Let $p_{\eta}=\bigcup_{i=0}^{\infty} p_{\eta}^{i}$. Note that $\max \left(\bar{\Omega}_{i}(\bar{z})\right)<\min \left(\bar{\Omega}_{i}(R(i))\right), i \leq$ $\max \left(\bar{\Omega}_{i}(R(i))\right)$ and hence $\max \left(\bar{\Omega}_{i}(\bar{z}) \cup \bar{\Omega}_{i}(R(i))\right) \leq L(\bar{R}(i+1))$. Therefore the first two induction

\footnotetext{
${ }^{5}$ This is not an error $-\max \left(\bar{\Omega}_{k}(\{j\})\right)$ is not intended.
} 
hypotheses hold. By observing that $p_{\eta}$ is an isomorphism between $\tau_{i}\left(\bar{\Omega}_{i}(R(i))\right)$ and $\tau_{i}\left(\bar{\Omega}_{i}(\bar{z})\right)$ it follows that the last induction hypothesis holds as well.

Now suppose that $H(a)=b$ and $b \in R(i)$ and $a \in i$. Then there is some $\bar{b} \in \bar{z}$ such that $\Delta_{\bar{\Omega}_{i}(R(i)), \bar{\Omega}_{i}(\bar{z})}\left(\Omega_{a}(\{b\})\right)=\Omega_{a}(\{\bar{b}\})$. Since $\max \left(\bar{\Omega}_{i}(\bar{z})\right)<\min \left(\bar{\Omega}_{i}(R(i))\right)$, it follows that $\max \left(\Omega_{a}(\{\bar{b}\})\right)<$ $\min \left(\Omega_{a}(\{b\})\right)$. By the fact that $b$ is the least integer satisfying 4.4 it follows that there is some $j \in C(a)$ such that $\tau_{a}(\{b\})=\tau_{a}(\{j\})$ and $\Delta_{\bar{\Omega}_{a}(\{j\})} \circ r_{\eta} \circ \Delta_{\bar{\Omega}_{a}(\{j\})}^{-1} \neq \Delta_{\bar{\Omega}_{a}(\{b\})} \circ r_{\eta} \circ \Delta_{\bar{\Omega}_{a}(\{b\})}^{-1}$ and, hence there is some $n$ such that

$$
\begin{gathered}
\left.r_{\eta} \circ \Delta_{\bar{\Omega}_{a}(\{j\})}^{-1}(n) \neq \Delta_{\bar{\Omega}_{a}(\{j\})}^{-1}\left(\Delta_{\bar{\Omega}_{a}(\{b\})} \circ r_{\eta} \circ \Delta_{\bar{\Omega}_{a}(\{b\})}^{-1}(n)\right)=\Delta_{\bar{\Omega}_{a}(\{b\}), \bar{\Omega}_{a}(\{j\})} \circ r_{\eta} \circ \Delta_{\bar{\Omega}_{a}(\{b\})}^{-1}(n)\right)= \\
\Delta_{\bar{\Omega}_{a}(\{b\}), \bar{\Omega}_{a}(\{j\})} \circ r_{\eta} \circ \Delta_{\bar{\Omega}_{a}(\{b\})}^{-1}\left(\Delta_{\bar{\Omega}_{a}(\{j\})} \circ \Delta_{\bar{\Omega}_{a}(\{j\})}^{-1}(n)\right)
\end{gathered}
$$

and therefore $r_{\eta}\left(\Delta_{\bar{\Omega}_{a}(\{j\})}^{-1}(n)\right) \neq\left(p_{\eta}^{i} \circ r_{\eta} \circ p_{\eta}^{i}\right)\left(\Delta_{\bar{\Omega}_{a}(\{j\})}^{-1}(n)\right)$. It follows that $p_{\eta} \circ r_{\eta}$ and $r_{\eta} \circ p_{\eta}$ disagree on infinitely many integers.

Notation 4.1. The notation $\mathbb{L}$ will be used to denote the Laver partial order. Most of the notation and terminology regarding this partial order will be taken from [6]. Let $E_{T}:{ }^{\omega} \omega \rightarrow T$ be the unique bijection preserving the lexicographic ordering from ${ }^{\omega} \omega$ onto the nodes above the root of $T$. If $T \in \mathbb{L}$ and $t \in{ }^{\omega} \omega$ let $T\langle t\rangle$ denote $E_{T}(t)$. If $s \in T$ then $T_{s}$ will denote the subtree of $T$ consisting all nodes comparable to $t$. Fix an enumeration $\left\{s_{n}\right\}_{n=0}^{\infty}$ of ${ }^{\omega} \omega$ such that if $s_{n} \subseteq s_{m}$ then $n \leq m$. Given $T \in \mathbb{L}$ and $n \in \mathbb{N}$ let $\left\{S_{n, j}(T)\right\}_{n=0}^{j}$ list the components 5 of $T$ determined by $\left\{s_{n}\right\}_{n \in j}$ - to be precise, $S_{n, j}(T)$ is the subtree of $T\left\langle s_{n}\right\rangle$ consisting of all nodes $t$ such that if $n<m \leq j$ then $s_{m} \not \subset t$.

The notation $\mathbb{L}_{\alpha}$ will be used to denote the countable support iteration of $\alpha$ Laver partial orders. For the definition of $p \geq_{F}^{n} q$ see $[6]$.

Lemma 4.3. If $p \in \mathbb{L}_{\alpha}, n \in \mathbb{N}$, $\stackrel{\circ}{m}$ is a $\mathbb{L}_{\alpha}$-name for an integer and $F$ is a finite subset of the domain of $p$ then there is some $p^{\prime}$ such that

- $p^{\prime}(0) \geq p(0)$

- $p^{\prime}(0)\langle 0\rangle=p(0)\langle 0\rangle$

- $p^{\prime} \uparrow[1, \alpha) \geq_{F}^{n} p\lceil[1, \alpha)$

- there is $U \subseteq \mathbb{N}$ such that $|U| \leq(n+1)^{|F|}$ and for every integer $k$, for all but finitely many immediate successors $t$ of $p(0)\langle 0\rangle$

$$
p^{\prime}(0)_{t} \smile p^{\prime} \uparrow[1, \alpha) \Vdash_{\mathbb{L}_{\alpha}} \text { “ } \stackrel{\circ}{m \in} U \cup(\mathbb{N} \backslash \check{k}) "
$$

Proof. If $n=0$ then this follows from Lemma 12 of [6]. Let $\stackrel{\circ}{a}$ of that Lemma be $\frac{1}{\circ}$ and find $u$ and $p^{\prime}$ such that for all $\epsilon>0$

$$
p^{\prime}(0)_{t} \frown p^{\prime} \uparrow[1, \alpha) \Vdash_{\mathbb{L}_{\alpha}} \text { “| } \stackrel{\circ}{a}-u \mid<\epsilon "
$$

for all but finitely many immediate successors $t$ of $p(0)\langle 0\rangle$. If $u \neq 0$ let $\epsilon$ be small enough that there is some $U=\{j\}$ such that $|u-1 / i|>\epsilon$ for all $i \geq 1$ unless $i=j$. Then for any immediate successor $t$ of $p(0)\langle 0\rangle$ such that $p^{\prime}(0)_{t} \smile p^{\prime} \uparrow[1, \alpha) \Vdash_{\mathbb{L}_{\alpha}}$ "| $\stackrel{\circ}{a}-u \mid<\epsilon$ " it follows that $p^{\prime}(0)_{t} \frown p^{\prime} \uparrow[1, \alpha) \Vdash_{\mathbb{L}_{\alpha}}$ " $\stackrel{\circ}{m \in U}$ " and hence

$$
p^{\prime}(0)_{t} \frown p^{\prime} \uparrow[1, \alpha) \Vdash_{\mathbb{L}_{\alpha}} \text { " } \stackrel{\circ}{m} \in U \text { ". }
$$

If $u=0$ and $k \in \mathbb{N}$ let $\epsilon<1 / k(k+1)$. Then for any immediate successor $t$ of $p(0)\langle 0\rangle$ such that $p^{\prime}(0)_{t} \frown p^{\prime} \uparrow[1, \alpha) \Vdash_{\mathbb{L}_{\alpha}} "|\stackrel{\circ}{a}-u|<\epsilon "$ it follows that $p^{\prime}(0)_{t} \frown p^{\prime} \uparrow[1, \alpha) \Vdash_{\mathbb{L}_{\alpha}}$ " $\stackrel{\circ}{m \in \mathbb{N} \backslash \breve{k} " . ~}$

The case for $n>0$ follows from the case $n=0$ by induction and a counting argument.

Lemma 4.4. For any ordinal $\alpha$ the partial order $\mathbb{L}_{\alpha}$ is weakly dominated over $V$.

Proof. Let $f: \mathbb{N} \rightarrow \mathbb{N}$ belong to $V$ and $H$ be an $\mathbb{L}_{\alpha}$-name for a function from $\mathbb{N}$ to $\mathbb{N}$. Let $p \in \mathbb{L}_{\alpha}$. The function $R \in \prod_{i=1}^{\infty}[\mathbb{N}] \leq i$ satisfying the three conditions of Definition 4.1 will be constructed by induction in $V$ unless $H$ is dominated by a function in $V$.

Let $\theta, \beta$ and $\delta$ be monotone functions from $\mathbb{N}$ to $\mathbb{N}$ such that

- $\lim _{n \rightarrow \infty} \theta(n)=\lim _{n \rightarrow \infty} \delta(n)=\lim _{n \rightarrow \infty} \beta(n)=\infty$

\footnotetext{
${ }^{6}$ See the definition on page 156 of $[6]$.
} 
- if $s_{m}$ is the predecessor or $s_{n}$ then $\beta(n) \leq \beta(m)+1$

- $\beta(n) \cdot(\theta(n)+1)^{\delta(n)} \leq n$

- $\beta(n)<n$.

Then construct $\left\{\left(p_{n}, F(n), R^{n}\right)\right\}_{n=0}^{\infty}$ by induction to satisfy the following conditions.

(1) $p_{0}=p$

(2) $p_{n} \leq_{F(n)}^{\theta(n)} p_{n+1}$

(3) $|F(n)|=\delta(n)$ and $F(n) \subseteq[1, \alpha)$

(4) $F(n+1) \supseteq F(n)$

(5) $p_{n}(0)\left\langle s_{i}\right\rangle=p_{n+1}(0)\left\langle s_{i}\right\rangle$ if $i \leq n$

(6) if $n \geq 1$ then for each $a \leq \beta(n)$ there is $U_{a}^{n} \subseteq \mathbb{N}$ such that

$$
R^{n}=\bigcup_{a \in \beta(n)} U_{a}^{n} \backslash f\left(\max \left(R^{n-1}\right)\right)
$$

(7) $\left|U_{a}^{n}\right| \leq(\theta(n)+1)^{\delta(n)}$ for each $a \leq \beta(n)$

(8) for all $m \leq n$ and $a \leq \beta(m)$ and $k \in \mathbb{N}$ and for all but finitely many $t$ which are immediate successors of $S_{m, n}\left(p_{n}(0)\right)\langle 0\rangle$

$$
S_{m, n}\left(p_{n}(0)\right)_{t} \frown p_{n} \uparrow[1, \alpha) \Vdash_{\mathbb{L}_{\alpha}} " H(\check{a}) \in \check{U}_{a}^{m} \cup(\mathbb{N} \backslash k) "
$$

(9) for all $m \leq n$ and $a \leq \beta(m)$

$$
S_{m, n}\left(p_{n}(0)\right)^{\frown} p_{n}\left\lceil[1, \alpha) \Vdash_{\mathbb{L}_{\alpha}} " H(\check{a}) \in \check{U}_{a}^{m} \cup\left(\mathbb{N} \backslash f\left(\max \left(R^{n}\right)\right)\right) " .\right.
$$

It will first be shown that this suffices. Let $p_{\omega}$ be the limit of the $p_{n}$ and define $R: \mathbb{N} \rightarrow[\mathbb{N}] \leq \aleph_{0}$ by letting $R(n)=R^{n}$ for each integer $n$. Let $f^{*}$ be defined by

$$
f^{*}(n)=f\left(\max \left(\bigcup_{\beta(i) \leq n} R^{i}\right)\right) .
$$

If $H \leq^{*} f^{*}$ then $H$ is dominated by a function in $V$ and there is nothing to do; so suppose that $H \mathbb{Z}^{*} f^{*}$. From Conditions 6 and $\mathbf{0}$ and the choice of $\beta, \theta$ and $\delta$ it follows that $R \in \prod_{n=1}^{\infty}[\mathbb{N}] \leq n$. Furthermore, from Condition 6 it follows that $f\left(\max \left(\bigcup_{i \in n} R(i)\right)\right)<\min (R(n))$ for each $n$. To see that $p_{\omega}$ forces the last requirement to be satisfied, suppose not and that $q \geq p_{\omega}$ and $K$ are such that $q \Vdash_{\mathbb{L}_{\alpha}}$ " $(\forall n \geq K) H \cap(n \times R(n))=\emptyset "$ ". Extend $q$ so that $q \Vdash_{\mathbb{L}_{\alpha}} " H(\check{a})=\breve{b} \geq \check{f}^{*}(\check{a})$ " for some integers $a \geq K$ and $b$ and so that $q(0)\langle 0\rangle=s_{m}$ where $\beta(m)>a$ and $b<f\left(\max \left(R^{m}\right)\right)$. Let $m^{\prime}$ be the least integer such that $s_{m^{\prime}} \prec s_{m}$ and $b<f\left(\max \left(R^{m^{\prime}}\right)\right)$ and let $m^{\prime \prime}$ be such that $s_{m^{\prime \prime}}$ is the predecessor of $s_{m^{\prime}}$. It follows that $b \geq f\left(\max \left(R^{m^{\prime \prime}}\right)\right)$. Also observe that, if $\beta\left(m^{\prime}\right) \leq a$ then $b \geq f^{*}(a)$ implies that $b \geq f\left(\max \left(R^{m^{\prime}}\right)\right)$ which is a contradiction. Hence, $\beta\left(m^{\prime}\right)>a$ and, by the hypothesis on $\beta, \beta\left(m^{\prime \prime}\right) \geq a$. If it could be established that $b \in U_{a}^{m^{\prime \prime}}$ it would follow that $(a, b) \in\left(\beta\left(m^{\prime \prime}\right)+1\right) \times R^{m^{\prime \prime}} \subseteq m^{\prime \prime} \times R\left(m^{\prime \prime}\right)$ and this would suffice.

There are now two cases to consider: Either $b<f\left(\max \left(R^{m^{\prime}-1}\right)\right)$ or $b \geq f\left(\max \left(R^{m^{\prime}-1}\right)\right)$. In the first case use Condition 9 to conclude that

$$
S_{m^{\prime \prime}, m^{\prime}-1}\left(p_{m^{\prime}-1}(0)\right) \frown p_{m^{\prime}-1}\left\lceil[1, \alpha) \Vdash_{\mathbb{L}_{\alpha}} " H(\check{a}) \in \check{U}_{a}^{m^{\prime \prime}} " .\right.
$$

Because $S_{m^{\prime \prime}, m^{\prime}-1}\left(p_{m^{\prime}-1}(0)\right)^{\frown} p_{m^{\prime}-1} \uparrow[1, \alpha) \leq q$ it follows that $b$ is not ruled out as a possible value for $H(\check{a})$ by $S_{m^{\prime \prime}, m^{\prime}-1}\left(p_{m^{\prime}-1}(0)\right)^{\frown} p_{m^{\prime}-1} \uparrow[1, \alpha)$ and, hence, that $b \in U_{a}^{m^{\prime \prime}}$ as required. In the second case, it would suffice to show that $b \in U_{a}^{m^{\prime}}$ because then it would follow that $(a, b) \in\left(\beta\left(m^{\prime}\right)+1\right) \times R\left(m^{\prime}\right)$. But this is clear since

$$
S_{m^{\prime}, m^{\prime}}\left(p_{m^{\prime}}(0)\right) \frown p_{m^{\prime}} \uparrow[1, \alpha) \leq q
$$

and so $b$ is not ruled out as a possible value for $H(\check{a})$ in this case either.

To show that the induction can be carried out, let $p_{0}=p$ and suppose that $\left\{\left(p_{n}, F(n), R^{n}\right)\right\}_{n \in j}$ have been constructed satisfying the required induction hypotheses. Let $F(j)$ be given by some bookkeeping scheme so that Conditions 3 and 1 are satisfied. Using Lemma 4.3 find $p$ such that

- $p(0) \geq S_{j, j}\left(p_{j-1}(0)\right)$

- $p(0)\langle 0\rangle=S_{j, j}\left(p_{j-1}(0)\right)\langle 0\rangle=p_{j-1}(0)\left\langle s_{j}\right\rangle$ 
- $p\left\lceil[1, \alpha) \geq_{F(j)}^{\theta(j)} p_{j-1}\lceil[1, \alpha)\right.$

- there are $U_{a}^{j} \subseteq \mathbb{N}$ for each $a \leq \beta(j)$ such that $\left|U_{a}^{j}\right| \leq(\theta(j)+1)^{|\delta(j)|}$ and for every integer $k$, for all but finitely many immediate successors $t$ of $p_{j-1}(0)\left\langle s_{j}\right\rangle$

$$
p(0)_{t} \frown p\left\lceil[1, \alpha) \Vdash_{\mathbb{L}_{\alpha}} " H(a) \in \check{U}_{a}^{j} \cup(\mathbb{N} \backslash \check{k}) " .\right.
$$

Then let $R^{j}$ be defined according to Condition 6 . Now, by removing finitely many immediate successors of $p_{j-1}(0)\left\langle s_{j}\right\rangle$ from $p(0)$ it is possible to obtain $\bar{p}_{j} \subseteq p(0)$ such that $\bar{p}_{j}\langle 0\rangle=p(0)\langle 0\rangle$ and for each $a \leq \beta(j)$

$$
\bar{p}_{j} \frown p\left\lceil[1, \alpha) \Vdash_{\mathbb{L}_{\alpha}} \text { " } H(a) \in \check{U}_{a}^{j} \cup\left(\mathbb{N} \backslash \max \left(R^{j}\right)\right) " .\right.
$$

Similarly, but using the induction hypothesis for each $n \leq j-1$ to remove finitely many immediate successors of $p_{j-1}(0)\left\langle s_{n}\right\rangle$ from $S_{n, j-1}\left(p_{j-1}(0)\right)$, it is possible to obtain $\bar{p}_{n} \subseteq S_{n, j-1}\left(p_{j-1}(0)\right)$ such that $\bar{p}_{n}\langle 0\rangle=p_{j-1}(0)\left\langle s_{n}\right\rangle$ and for each $a \leq \beta(n)$

$$
\bar{p}_{n} \frown p_{j-1} \uparrow[1, \alpha) \Vdash_{\mathbb{L}_{\alpha}} " H(a) \in \check{U}_{a}^{n} \cup\left(\mathbb{N} \backslash \max \left(R^{j}\right)\right) " .
$$

Let

$$
p_{j}^{*}=\bar{p}_{j} \frown p\lceil[1, \alpha)
$$

and, for $n \leq j-1$, let

$$
p_{n}^{*}=\bar{p}_{n} \frown p_{j-1} \uparrow[1, \alpha)
$$

and then define $p_{j}$ to be the join of $\left\{p_{i}^{*}\right\}_{i=0}^{j}$. It is immediate to check that all of the induction hypotheses are satisfied by $p_{j}, F(j)$ and $R^{j}$.

The following result, due to S. Shelah, is 5.31 in [12]. It will be useful to know that Laver forcing $\mathbb{L}$ is NEP.

Lemma 4.5. Let $\left\{B_{\alpha}\right\}_{\alpha \in \omega_{1}}$ be family of Borel sets in the model of set theory $V$ such that $V \models$ $\bigcap_{\alpha \in \omega_{1}} B_{\alpha}=\emptyset$. Let $\mathbb{P}$ be a NEP partial order with definition in $V$ and suppose that $\left\{\mathbb{P}_{\alpha}\right\}_{\alpha \in \omega_{2}}$ is a countable support iteration such that $\mathbb{P}_{\alpha+1}=\mathbb{P}_{\alpha} * \mathbb{P}$ for any $\alpha \in \omega_{2}$. If

$$
1 \Vdash_{\mathbb{P}_{\alpha+1}} " \bigcap_{\alpha \in \omega_{1}} B_{\alpha}=\emptyset "
$$

for each $\alpha \in \omega_{1}$ then

$$
1 \Vdash_{\mathbb{P}_{\omega_{2}}} " \bigcap_{\alpha \in \omega_{1}} B_{\alpha}=\emptyset " .
$$

Theorem 4.1. It is consistent that $A\left([\mathbb{N}]^{<\aleph_{0}}\right)=\aleph_{1}<\mathfrak{a}$.

Proof. The model witnessing this is the one obtained by forcing with $\mathbb{L}_{\omega_{2}}$ over a model $V$ satisfying $2^{\aleph_{0}}=\aleph_{1}$. From Lemma 4.2 and Lemma 4.4 it follows that there are permutations $\left\{p_{\xi}\right\}_{\xi \in \omega_{1}}$ of $\mathbb{N}$ in $V$ which mutually almost commute and which are maximal with respect to this property; in other words, letting $B_{\alpha}$ be the Borel set of all permutations of the integers which almost commute with $p_{\alpha}$ but are not equal to $p_{\alpha}$ modulo a finite set, $\bigcap_{\alpha \in \omega_{1}} B_{\alpha}=\emptyset$. Moreover, it follows from Lemma 4.2 and Lemma 4.4 that

$$
1 \Vdash_{\mathbb{L}_{\omega_{1}}} " \bigcap_{\alpha \in \omega_{1}} B_{\alpha}=\emptyset "
$$

and, hence by Lemma 4.5 it follows that

$$
1 \Vdash_{\mathbb{L}_{\omega_{2}}} " \bigcap_{\alpha \in \omega_{1}} B_{\alpha}=\emptyset "
$$

or, in other words, $1 \Vdash_{\mathbb{L}_{\omega_{2}}}$ "A([N] $\left.]^{<\aleph_{0}}\right)=\aleph_{1} "$.

The fact that $\mathfrak{a}=\aleph_{2}$ in this model is well known and can be found, for example, in [2]. 


\section{The Cohen model And the summable ideals}

The ideal $\mathcal{I}_{1 / x}$ is defined to be the set of all $X \subseteq \mathbb{N}$ such that $\sum_{x \in X} 1 / x<\infty$. It will be shown that $\mathbb{S}\left(\mathcal{I}_{1 / x}\right) / \mathbb{F}\left(\mathcal{I}_{1 / x}\right)$ has a maximal Abelian subgroup of size $\aleph_{1}$ in any model obtained by adding uncountably many Cohen reals for any function $h$ such that $h(n) \geq 1 / n$ for all but finitely many $n \in \mathbb{N}$. The basic scheme of the argument is that in a model of the form $V\left[\left\{c_{\xi}\right\}_{\xi \in \omega_{1}}\right]$ where $\left\{c_{\xi}\right\}_{\xi \in \omega_{1}}$ are Cohen reals, it is possible to define permutations $\left\{\pi_{\xi}\right\}_{\xi \in \omega_{1}}$ such that $\pi_{\xi} \in V\left[\left\{c_{\eta}\right\}_{\eta \in \xi+1}\right]$ which form an Abelian subgroup which is close to maximal in the following sense: If $G$ is the group generated by $\left\{\pi_{\xi}\right\}_{\xi \in \omega_{1}}, G^{\prime}$ is the group generated by $G$ and all permutations which are first order definable from $G$ and $G^{\prime \prime}$ is a maximal Abelian subgroup of $G^{\prime}$ containing $G$ then $G^{\prime \prime}$ is actually a maximal Abelian subgroup. Consequently, for most of the rest of this section a family of permutations with certain properties will be fixed - these should thought of as the permutations obtained from the Cohen reals. Some notation will first be established.

Suppose that $\left\{\pi_{\xi}\right\}_{\xi \in \alpha}$ have been constructed. Let $\left\{\alpha_{i}\right\}_{i=0}^{\infty}$ enumerate $\alpha$ and, by re-indexing, suppose that $\pi_{n}=\pi_{\alpha(n)}$. Let $\Omega_{m}=\Omega_{\left\{\pi_{i}\right\}_{i \in n}}$. The elements of $\Omega_{m}$ will be enumerated as $\left\{\Omega_{m}^{i}\right\}_{i=0}^{\infty}$ in such a way that $i<j$ if and only if $\min \left(\Omega_{m}^{i}\right)<\min \left(\Omega_{m}^{j}\right)$. The following technical definition will be the key to the induction hypothesis of the construction.

Definition 5.1. A family of permutations $\mathcal{F}=\left\{\pi_{n}\right\}_{n=0}^{\infty}$ will be said to be nice if

(1) $\lim _{n \rightarrow \infty} \frac{\pi_{k}(n)}{n}=1$ for each $k$

(2) $\pi_{k}=\pi_{k}^{-1}$ for each $k$

(3) $\pi_{k}$ and $\pi_{m}$ almost commute for each $k$ and $m$

(4) for every $m \in \mathbb{N}$ there is an integer $K_{m}$ such that if $i$ and $j$ are greater than $K_{m}$ then $\Omega_{m}^{i}$ and $\Omega_{m}^{j}$ are $\left\{\pi_{i}\right\}_{i \in m}$-isomorphic. (See Definition 1.5)

Definition 5.2. Given a nice family $\mathcal{F}$ define the partial order $\mathbb{P}(\mathcal{F})$ to consist of triples $p=$ $\left(f_{p}, m_{p}, \epsilon_{p}\right)$ such that $m_{p} \in \mathbb{N}, \epsilon_{p}>0$ and there is an integer $I_{p}$ such that $f_{p}$ is a permutation of $\Omega_{m_{p}}\left(I_{p}\right)$ such that $f_{p}^{-1}=f_{p}$ and

$$
\left(\forall n \in \mathbb{N} \backslash \operatorname{domain}\left(f_{p}\right)\right)\left(\forall i \leq m_{p}\right)\left(\forall j \leq m_{p}\right) \pi_{i} \circ \pi_{j}(n)=\pi_{j} \circ \pi_{i}(n)
$$

and if

$$
\delta=\sup _{j \in \mathbb{N} \backslash \text { domain }\left(f_{p}\right)}\left(\sup _{n \leq m_{p}}\left|1-\frac{\pi_{n}(j)}{j}\right|\right)
$$

then

$$
\left(1+2^{m}\left(1-\frac{1}{(1+\delta)^{m}}\right)\right)(1+\delta)^{m}<1+\epsilon_{p} .
$$

Define $p \leq q$ if $\epsilon_{p} \leq \epsilon_{q}, m_{p} \geq m_{q}$ and

$$
f_{p}=f_{q} \cup \bigcup_{\{u, v\} \in t} \Delta_{\Omega_{m_{p}}^{u}, \Omega_{m_{p}}^{v}}
$$

where $t$ partitions $I_{p} \backslash I_{q}$ into pairs, and

$$
1-\epsilon_{q}<\frac{f_{p}(i)}{i}<1+\epsilon_{q}
$$

for each $i$ in the domain of $f_{p} \backslash f_{q}$.

Lemma 5.1. Let $\mathcal{F}$ be a nice family, $m \in \mathbb{N}$ and $K_{m}$ be as in Definition 5.1 and suppose that $i$ and $j$ are both greater than $K_{m}$. Suppose further that $k \geq i$ and $x \in \Omega_{m}^{i}$ then

$$
(1-\epsilon)<\frac{\pi_{n}(x)}{x}<(1+\epsilon)
$$


for each $n \in m$. Then the following inequalities hold:

$$
\begin{gathered}
\frac{\max \left(\Omega_{m}^{i}\right)}{\min \left(\Omega_{m}^{i}\right)}<(1+\epsilon)^{m} \\
\frac{\min \left(\Omega_{m}^{i+1}\right)}{\min \left(\Omega_{m}^{i}\right)}<1+2^{m}\left(1-\frac{1}{(1+\epsilon)^{m}}\right) \\
\frac{\min \left(\Omega_{m}^{i+k}\right)}{\min \left(\Omega_{m}^{i}\right)}<\left(1+2^{m}\left(1-\frac{1}{(1+\epsilon)^{m}}\right)\right)^{k}
\end{gathered}
$$

and, furthermore, if $\Phi: \Omega_{m}^{i} \rightarrow \Omega_{m}^{i+k}$ is a $\left\{\pi_{i}\right\}_{i \in m}$-isomorphism then

$$
(1-\epsilon)^{m}<\frac{\Phi(n)}{n}<\left(1+2^{m}\left(1-\frac{1}{(1+\epsilon)^{m}}\right)\right)^{k}(1+\epsilon)^{m}
$$

Proof. In order to prove 5.7 the first thing to note is that if $a \in \Omega_{m}$ and $\{x, y\} \subseteq a$ then there is $k \leq m$ and a sequence $\left(u_{1}, u_{2}, \ldots, u_{k}\right) \in m^{k}$ such that

$$
x=\pi_{u_{1}} \circ \pi_{u_{2}} \circ \ldots \circ \pi_{u_{k}}(y) .
$$

Given $x \in \Omega_{m}^{i}$ let $k(x)$ be the least integer such that

$$
x=\pi_{u_{1}} \circ \pi_{u_{2}} \circ \ldots \circ \pi_{u_{k(x)}}\left(\min \left(\Omega_{m}^{i}\right)\right)
$$

and proceed by induction on $k(x)$. If $k(x)=0$ then $x=\min \left(\Omega_{m}^{i}\right)$ and the result is clear. Suppose that the lemma has been established for all $x$ such that $k(x)=n$. Given $x$ such that $k(x)=n+1$ it is possible to find $x^{\prime}$ such that $k\left(x^{\prime}\right)=n$ and $x=\pi_{u}\left(x^{\prime}\right)$ for some $u \in m$. From 5.6 it follows that

$$
(1-\epsilon)<\frac{\pi_{u}\left(x^{\prime}\right)}{x^{\prime}}<(1+\epsilon)
$$

and from the induction hypothesis it follows that

$$
(1-\epsilon)^{n}<\frac{x^{\prime}}{\min \left(\Omega_{m}^{j}\right)}<(1+\epsilon)^{n} .
$$

Hence,

as desired.

$$
(1-\epsilon)^{n+1}<\frac{x}{\min \left(\Omega_{m}^{j}\right)}<(1+\epsilon)^{n+1}
$$

To see that 5.8 holds begin by observing that if $i^{\prime}<i$ and $\Omega_{m}^{i^{\prime}} \backslash \min \left(\Omega_{m}^{i}\right) \neq \emptyset$ then, by 5.7 ,

$$
\min \left(\Omega_{m}^{i}\right)<\max \left(\Omega_{m}^{i^{\prime}}\right)<\min \left(\Omega_{m}^{i^{\prime}}\right)(1+\epsilon)^{m}
$$

and hence

Therefore, the cardinality of

$$
\min \left(\Omega_{m}^{i^{\prime}}\right)>\frac{\min \left(\Omega_{m}^{i}\right)}{(1+\epsilon)^{m}}
$$

$$
\bigcup_{i^{\prime} \leq i} \Omega_{m}^{i^{\prime}} \backslash \min \left(\Omega_{m}^{i}\right)
$$

is no greater than

$$
\left(\min \left(\Omega_{m}^{i}\right)-\frac{\min \left(\Omega_{m}^{i}\right)}{(1+\epsilon)^{m}}\right) 2^{m}
$$

and it follows that

$$
\min \left(\Omega_{m}^{i+1}\right)<\min \left(\Omega_{m}^{i}\right)+\min \left(\Omega_{m}^{i}\right)\left(1-\frac{1}{(1+\epsilon)^{m}}\right) 2^{m}
$$

as required.

The general statement 5.9 follows by repeated application of 5.8 .

To prove 5.10 let $n \in \Omega_{m}^{i}$. Combining 5.7 and 5.9 yields

$$
\frac{\Phi(n)}{n} \leq \frac{\min \left(\Omega_{m}^{i}\right)}{\min \left(\Omega_{m}^{i}\right)}<\left(1+2^{m}\left(1-\frac{1}{(1+\epsilon)^{m}}\right)\right)^{k}(1+\epsilon)^{m}
$$


establishing the last half of the inequality. For the first half note that $\min \left(\Omega_{m}^{i}\right) \leq \min \left(\Omega_{m}^{i+k}\right)$ and hence, from 5.7 and 5.9 it follows that

$$
\frac{n}{\Phi(n)} \leq \frac{\max \left(\Omega_{m}^{i}\right)}{\min \left(\Omega_{m}^{i}\right)} \frac{\min \left(\Omega_{m}^{i}\right)}{\min \left(\Omega_{m}^{i+k}\right)}>\frac{1}{(1+\epsilon)^{m}} \geq(1-\epsilon)^{m} .
$$

Lemma 5.2. If $p \in \mathbb{P}(\mathcal{F})$ and $u \in \mathbb{N}$ then there is $f$ such that $\left(f, m_{p}, \epsilon_{p}\right) \leq p$ and $u \in \operatorname{domain}(f)$.

Proof. It suffices to prove this for the case that $u$ is the least integer not in the domain of $f_{p}$. Let $i=I_{p}$ and let $f=f_{p} \cup \Phi \cup \Phi^{-1}$ where $\Phi: \Omega_{m}^{i} \rightarrow \Omega_{m}^{i+1}$ is an isomorphism. From Condition 5.3 of Definition 5.2 and Conclusion 5.10 of Lemma 5.1, with $k=1$ it follows that Requirement 5.5 is satisfied. Since requirement 5.4 is immediate, this suffices.

Lemma 5.3. If $p \in \mathbb{P}(\mathcal{F})$ and $\epsilon>0$ and $m \in \mathbb{N}$ then there is $q \leq p$ such that $\epsilon_{q} \leq \epsilon$ and $m_{q} \geq m$.

Proof. First apply Lemma 5.2 to extend $p$ to $\left(f, m_{p}, \epsilon_{p}\right)$ such that the domain of $f$ is sufficiently large that it is possible to change $m_{p}$ to $m$ and $\epsilon_{p}$ to $\epsilon$ and still preserve Condition 5.1, 5.2 and 5.3.

Lemma 5.4. If $p \in \mathbb{P}(\mathcal{F})$ and $\pi \in \mathbb{S}\left(\mathcal{I}_{1 / x}\right)$ but $[\pi]_{\mathcal{I}_{1 / x}}$ is not first order definable from $\left\{\pi_{i}\right\}_{i=0}^{\infty} / \mathbb{F}\left(\mathcal{I}_{1 / x}\right)$ and $k \in \mathbb{N}$ then there is $q \leq p$ such that $\sum_{i=k}^{\infty}\left\{h(i): \pi\left(f_{q}(i)\right) \neq f_{q}(\pi(i))\right\}>1$.

Proof. As a convenience, let $m=m_{p}$ and $J=I_{p}$. From the definition of $J$ it follows that it is possible to choose a family of mappings $\left\{\Phi_{i, j}\right\}_{i, j \geq J}$ such that $\Phi_{i, j}: \Omega_{m}^{i} \rightarrow \Omega_{m}^{j}$ is an isomorphism, $\Phi_{i, j} \circ \Phi_{j, i}$ is the identity on $\Omega_{m}^{i}$ and $\Phi_{i, k} \circ \Phi_{j, i}=\Phi_{j, k}$. Also, by appealing to Lemma 5.2 it may assumed that if

$$
\delta=\sup _{j \in \mathbb{N} \backslash \operatorname{domain}\left(f_{p}\right)}\left(\sup _{n \leq m_{p}}\left|1-\frac{\pi_{n}(j)}{j}\right|\right)
$$

then

$$
\left(1+2^{m}\left(1-\frac{1}{(1+\delta)^{m}}\right)\right)^{6}(1+\delta)^{m}<1+\epsilon_{p}
$$

The following fact will play a role later in the proof but is included here to explain the significance of the exponent 6 in 5.11 as well as in the indexing to follow.

Claim 4. Given any $\pi \in \operatorname{Sym}(6)$ other than the identity there is $\sigma \in \operatorname{Sym}(6)$ without fixed points such that $\sigma$ is an involution and $\sigma$ does not commute with $\pi$.

Define $E_{i}=\bigcup_{w=0}^{5} \Omega_{m}^{J+6 i+w}$ for each $i \in \mathbb{N}$. For $\sigma$ an involution in Sym(6) and integers $i$ and $j$ let $H_{i, j}^{\sigma}: E_{i} \rightarrow E_{j}$ be the isomorphism defined by

$$
H_{i, j}^{\sigma}=\bigcup_{w=0}^{5} \Phi_{J+6 i+w, J+6 j+\sigma(w)} \cup \Phi_{J+6 j \sigma(w), J+6 i+w} .
$$

If $\sigma$ is the identity then $H_{i, j}^{\sigma}$ will be denoted by $H_{i, j}$ and if $i=j$ then $H_{i j}^{\sigma}$ will be denoted by $H_{i}^{\sigma}$.

Observe that if $X \subseteq \mathbb{N}$ and $y_{i} \in E_{i}$ for $i \in X$ then, by 5.10 of Lemma 5.1,

$$
\sum_{i \in X} 1 / y_{i}<\infty \text { if and only if } \sum_{i \in X} \sum_{y \in E_{i}} 1 / y<\infty .
$$

This will be used repeatedly in order to restrict the possible structure of $\pi$.

To begin, let $W(x)$ be defined so that if $x \in \Omega_{m}^{i}$ then $\pi(x) \in \Omega_{m}^{W(i)}$. First note that if there exist $x_{1}$ and $x_{2}$ in $\Omega_{m}^{u}$ such that $W\left(x_{1}\right) \neq W\left(x_{2}\right)$ then there exists a sequence $\left(u_{1}, u_{2}, \ldots, u_{k}\right)$ of integers in $m$ such that

$$
x_{2}=\pi_{u_{1}} \circ \pi_{u_{2}} \circ \ldots \pi_{u_{k}}\left(x_{1}\right)
$$

and, hence, $\pi\left(\pi_{u_{1}} \circ \pi_{u_{2}} \circ \ldots \pi_{u_{k}}\left(x_{1}\right)\right)=\pi\left(x_{2}\right) \neq \pi_{u_{1}} \circ \pi_{u_{2}} \circ \ldots \pi_{u_{k}}\left(\pi\left(x_{1}\right)\right)$ because the elements of $\Omega_{m}$ are closed under the permutations $\pi_{u_{i}}$. Therefore, by 5.12, it may be assumed that if $Z$ is the set of all $i$ such that there is a pair $\{x, y\} \subseteq a \in \Omega_{m}$ and $a \subseteq E_{i}$ and $W(x) \neq W(y)$ then $\sum_{i \in Z} \sum_{y \in E_{i}} 1 / y<\infty$.

Next, let $W^{\prime}$ be defined such that if $x \in E_{i}$ then $\Omega_{m}^{W(x)} \in E_{W^{\prime}(x)}$. Let

$$
X=\left\{i \in \mathbb{N}:\left(\exists y \in E_{i}\right)\left(\exists x \in E_{i}\right) W^{\prime}(y) \neq W^{\prime}(x)\right\}
$$


and suppose that $\sum_{i \in X} \sum_{y \in E_{i}} 1 / y=\infty$. Then, using 5.12, it is possible to choose a finite $A \subseteq X$ such that if $y_{a} \in E_{a}$ and $x_{a} \in E_{a}$ are chosen for each $a \in A$ such that $W^{\prime}\left(y_{a}\right) \neq W^{\prime}\left(x_{a}\right)$ then $\sum_{a \in A} 1 / y_{a}>1$. Let $\sigma_{a}$ be a fixed point free involution of 6 such that if $y_{a} \in \Omega_{m}^{J+6 i+w_{y}}$ and $x_{a} \in \Omega_{m}^{J+6 i+w_{x}}$ then $\sigma_{a}\left(w_{y}\right)=w_{x}$. It follows that if $q \leq p$ is the condition defined by

$$
f_{q}=f_{p} \cup \bigcup_{a \in A} H_{a}^{\sigma_{a}}
$$

then, by 5.11 and Conclusion 5.10 of Lemma 5.1, it follows that Condition 5.3 of Definition 5.2 is satisfied. Since each $\sigma_{a}$ is fixed point free it follows that Condition 5.4 of Definition 5.2 is also satisfied. Therefore $q \leq p$ and it follows from the choice of $A$ that $q$ satisfies the requirements of the lemma.

Hence, it may be assumed that there is a set $Y$ such that $\bigcup_{i \in \mathbb{N} \backslash Y} E_{i} \in \mathcal{I}_{1 / x}$ and $W^{\prime}$ is constant on $E_{i}$ for each $i \in Y$. Let $W^{\prime \prime}$ be defined on $Y$ such that if $x \in E_{i}$ then $\Omega_{m}^{W^{\prime}(x)} \subseteq E_{W^{\prime \prime}(i)}$. Therefore there is a partition $Y=Y_{0} \cup Y_{1} \cup Y_{2} \cup Y_{4}$ such that $W^{\prime \prime}\left(Y_{i}\right) \cap Y_{i}=\emptyset$ for each $i \in 3$ and $W^{\prime \prime}$ is the identity on $Y_{4}$. Let $j \in 4$ be such that $\sum_{i \in Y_{j}} \sum_{y \in E_{i}} 1 / y=\infty$.

First assume that $j \in 3$. Let $A \subseteq Y_{j}$ be such that $\sum_{a \in A} 1 / y_{a}>1$ for any choice of $y_{a} \in E_{a}$ and let $B$ be the image of $A$ under $W^{\prime \prime}$. Choose involutions without fixed points $\sigma_{a} \in \operatorname{Sym}(6)$ and $\beta_{a} \in \operatorname{Sym}(6)$ for $a \in A$ such that

$$
H_{W^{\prime \prime}(a)}^{\beta_{a}} \circ \pi \uparrow E_{a} \neq \pi \circ H_{a}^{\sigma_{a}}
$$

and let $q \leq p$ be defined so that

$$
f_{q}=f_{p} \cup \bigcup_{a \in A} H_{a}^{\sigma_{a}} \cup H_{W^{\prime \prime}(a)}^{\beta_{a}}
$$

noting that $q \leq p$ as in the previous case.

Hence, assume that $j=3$. For $i \in Y_{3}$ let $W_{i} \in \operatorname{Sym}(6)$ be the permutation defined by $W(x) \in$ $\Omega_{m}^{W_{i}(w)}$ if $x \in \Omega_{m}^{J+6 i+w}$. If the set of $i \in Y_{3}$ such that $W_{i}$ is not the identity is not in $\mathcal{I}_{1 / x}$ then using Claim 1 it is possible to choose an involution $\sigma_{i} \in \operatorname{Sym}(6)$ without fixed points such that $\sigma^{i}$ does not commute with $W_{i}$. As before, using 5.12 it is possible to find a finite subset $A \subseteq Y_{3}$ such that setting $f_{q}=f_{p} \cup \bigcup_{a \in A} H_{a}^{\sigma_{a}}$ suffices.

Therefore, by omitting a set in $\mathcal{I}_{1 / x}$, it may be assumed that $W_{i}$ is the identity for all $i \in Y_{3}$ and that $\bigcup_{i \in \mathbb{N} \backslash Y_{3}} E_{i} \in \mathcal{I}_{1 / x}$. For $\rho \in \operatorname{Sym}\left(\Omega_{m}^{J}\right)$ and $z \in 6$ let $Y(\rho, z)$ be the set of all $i \in Y_{3}$ such that

$$
\Phi_{J+6 i+z, J} \circ \pi \circ \Phi_{J, J+6 i+z} \uparrow \Omega_{m}^{J}=\rho .
$$

If for each $z \in 6$ there is only one $\rho_{z} \in \operatorname{Sym}\left(\Omega_{m}^{J}\right)$ such that $\sum_{i \in Y\left(\rho_{z}, z\right)} \sum_{x \in \Omega_{m}^{i}} 1 / x=\infty$ then $[\pi]_{\mathcal{I}_{1 / x}}$ can be defined from $\left\{\pi_{j}\right\}_{j \in m}$ and $\left\{\rho_{z}\right\}_{z \in 6}$. So it may be assumed that it is possible to choose $z \in 6$ and $\rho_{z} \in \operatorname{Sym}\left(\Omega_{m}^{J}\right)$ such that if $U_{0}=Y\left(\rho_{z}, z\right)$ and $U_{1}=\mathbb{N} \backslash U_{0}$

$$
\sum_{i \in U_{0}} \sum_{x \in \Omega_{m}^{i}} 1 / x=\sum_{i \in U_{1}} \sum_{x \in \Omega_{m}^{i}} 1 / x=\infty .
$$

The key point to keep in mind is that if $i \in U_{0}$ and $j \in U_{1}$ and $q \leq p$ and $H_{i, j} \subseteq f_{q}$ then there is $x \in E_{i}$ such that $f_{q}(\pi(x)) \neq \pi\left(f_{q}(x)\right)$. Of course, if $|i-j|$ is too large then there might not be any $q$ such that $H_{i, j} \subseteq f_{q}$. The remainder of the argument is devoted to showing that there are sufficiently many pairs $(i, j) \in U_{0} \times U_{1}$ such that $f_{p}$ can be extended by $H_{i, j}$.

To this end, let $U=\left\{n \in U_{0}: n+1 \in U_{1}\right\}$. For $n \in U$ let $n_{0}$ be the greatest integer such that the interval $\left[n-n_{0}, n\right]$ is contained in $U_{0}$ and let $n_{1}$ be the largest integer such that $\left[n+1, n+1+n_{1}\right] \subseteq U_{1}$. Let $U_{0}^{*}$ be the set of all $n \in U$ such that $n_{0} \leq n_{1}$ and $U_{1}^{*}$ be the set of all $n \in U$ such that $n_{0}>n_{1}$ and define $U_{i}^{\prime}=\bigcup_{n \in U_{i}^{*}}\left[n_{0}, n_{1}\right]$ and observe that $Y_{3}=U_{0}^{\prime} \cup U_{1}^{\prime}$. Hence, either $U_{0}^{\prime}$ or $U_{1}^{\prime}$ belongs to $\mathcal{I}_{1 / x}$ In either case the following argument is similar so assume that the former holds.

Let $\bar{\epsilon}$ be so small that

and choose $\delta$ small enough that

$$
\frac{1}{1-2^{m} 6 \bar{\epsilon}}<1+\epsilon
$$

$$
\frac{\left(1+2^{m} J\left((1+\delta)^{6 m}-1\right)\right)(1+\delta)^{6 m}}{1-2^{m} 6\left(\bar{\epsilon}+(1+\delta)^{6 m}-1\right)} .
$$


Using Lemma 5.2, is may be assumed that

$$
1-\delta<\frac{\pi_{i}(x)}{x}<1+\delta
$$

for all $x \notin \operatorname{domain}\left(f_{p}\right)$ and $i \in m$.

There are again two cases to consider: Either there are infinitely many $n \in U_{0}^{\prime}$ such that $n_{0} \leq$ $(1-\bar{\epsilon}) n$ or there are not. In either case let $k_{n}=\max \left(n-n_{0},\lceil n(1-\bar{\epsilon})\rceil\right)$. Keep in mind that $\min \left(E_{i}\right)=\min \left(\Omega_{m}^{J+6 i}\right)$. Begin by observing, using Conclusion 5.7, that if $\Omega_{m}^{j}$ intersects the interval $\left[\left\lceil\min \left(E_{k_{n}}\right)(1+\delta)^{6 m}\right\rceil, \min \left(E_{n}\right)\right]$ then $J+6 k_{n} \leq j \leq J+6 n$. Hence,

$$
\left[\left\lceil\min \left(E_{k_{n}}\right)(1+\delta)^{6 m}\right\rceil, \min \left(E_{n}\right)\right] \subseteq \bigcup_{z=k_{n}}^{n} E_{z} \subseteq \bigcup_{z=\lceil n(1-\bar{\epsilon})\rceil}^{n} E_{z} \subseteq \bigcup_{z \in U_{i}^{\prime}} E_{z}
$$

for $n \in U_{0}^{\prime}$. Furthermore,

$$
\min \left(E_{n}\right)-\left\lceil\min \left(E_{k_{n}}\right)(1+\delta)^{6 m}\right\rceil \leq 2^{m} 6\left(n-k_{n}\right)
$$

It follows that

$$
\begin{gathered}
\min \left(E_{n}\right)-\min \left(E_{k_{n}}\right) \leq 2^{m} 6\left(n-k_{n}\right)+\left(\left\lceil\min \left(E_{k_{n}}\right)(1+\delta)^{6 m}\right\rceil-\min \left(E_{k_{n}}\right)\right) \\
\leq 2^{m} 6\left(n-k_{n}\right)+2^{m}\left(J+6 k_{n}\right)\left((1+\delta)^{6 m}-1\right) \\
\leq 2^{m}\left(6\left(n-k_{n}\right)+6 k_{n}\left((1+\delta)^{6 m}-1\right)\right)+2^{m} J\left((1+\delta)^{6 m}-1\right) \\
\leq 2^{m}\left(6\left(n-k_{n}\right)+6 n\left((1+\delta)^{6 m}-1\right)\right)+2^{m} J\left((1+\delta)^{6 m}-1\right) \\
\leq 2^{m} 6 n\left(\bar{\epsilon}+(1+\delta)^{6 m}-1\right)+2^{m} J\left((1+\delta)^{6 m}-1\right)
\end{gathered}
$$

and hence,

$$
\frac{\min \left(E_{n}\right)}{\min \left(E_{k_{n}}\right)}-1 \leq \frac{n}{\min \left(E_{k_{n}}\right)}\left(2^{m} 6\left(\bar{\epsilon}+(1+\delta)^{6 m}-1\right)\right)+2^{m} J\left((1+\delta)^{6 m}-1\right) .
$$

Therefore, using the fact that $n \leq \min \left(E_{n}\right)$,

$$
\frac{\min \left(E_{n}\right)}{\min \left(E_{k_{n}}\right)} \leq \frac{1+2^{m} J\left((1+\delta)^{6 m}-1\right)}{1-2^{m} 6\left(\bar{\epsilon}+(1+\delta)^{6 m}-1\right)} \leq \frac{1+\epsilon}{(1+\delta)^{6 m}}
$$

and so, using Conclusion 5.7.

$$
\frac{H_{k_{n}, n}(x)}{x} \leq \frac{\max \left(E_{n}\right)}{\min E_{k_{n}}} \leq 1+\epsilon .
$$

Similar reasoning shows that

$$
\frac{H_{i, i+k_{n}}(x)}{x} \leq 1+\epsilon
$$

for each $i$ such that $k_{n} \leq i<n$. Therefore extending $f_{p}$ to $f_{q}$ so that $f_{q}=f_{p} \cup \bigcup_{i=k_{n}}^{n} H_{i, i+k_{n}}$ will satisfy Conditions 5.4 and 5.5 of Definition 5.2 .

The only question which remains is whether it is possible to add enough of these extensions to provide a large witness to $f_{q}$ not commuting with $\pi$. In the case there is some $K$ such that $k_{n}=n_{0}$ for all $u \geq K$ it follows that $\sum_{n \in U_{0}^{\prime} \backslash K} \sum_{j=n_{0}}^{n} 1 / j=\infty$. Moreover, for each $n \geq K$ and $j$ such that $n_{0} \leq j \leq n$ there is some $x_{j} \in E_{j}$ such that $\pi\left(H_{j, j+n_{0}}\left(x_{j}\right)\right) \neq H_{j, j+n_{0}}\left(\pi\left(x_{j}\right)\right)$. Hence, by 5.12, it follows that $\sum_{j \in U_{0}^{\prime} \backslash K} 1 / x_{j}=\infty$ and so it is possible to choose $M$ such that defining $f_{q}=f_{p}=\bigcup_{n=K}^{M} \bigcup_{i=n_{0}}^{n} H_{i, i+k_{n}}$ works.

In the other case, there is an infinite set $U^{\prime \prime} \subseteq U_{0}^{\prime}$ such that $k_{n}=\lceil n(1-\bar{\epsilon})\rceil$ for each $n \in U^{\prime \prime}$. By 5.13 it follows that if $n \in U^{\prime \prime}$ then if $j \in\lceil u(1-\bar{\epsilon})\rceil \leq j \leq u$ then there is some $x_{j} \in E_{j}$ such that $\pi\left(H_{j, j+n_{0}}\left(x_{j}\right)\right) \neq H_{j, j+n_{0}}\left(\pi\left(x_{j}\right)\right)$. It follows that for $n \in U^{\prime \prime}$

$$
\begin{aligned}
\sum_{i=\lceil n(1-\bar{\epsilon})\rceil}^{n} 1 / x_{i} \geq & \sum_{i=\lceil n(1-\bar{\epsilon})\rceil}^{n} \frac{1}{\max \left(E_{i}\right)} \geq \sum_{i=\lceil n(1-\bar{\epsilon})\rceil}^{n} \frac{1}{\min \left(\Omega_{m}^{J+6 i+5}\right)(1+\delta)^{m}} \\
& \geq \sum_{i=\lceil n(1-\bar{\epsilon})\rceil}^{n} \frac{1}{2^{m}(J+6 i+5)(1+\delta)^{m}}
\end{aligned}
$$


and elementary calculations show that the limit as $n$ increases to infinity of the last term of the inequality is

$$
\frac{1}{2^{m} 6(1+\delta)^{m}} \ln \left(\frac{1}{1-\bar{\epsilon}}\right)=\gamma>0
$$

Now it suffices to choose a finite subset $X \subseteq U^{\prime \prime}$ such that

$$
|X|>1 / \gamma
$$

and define $f_{q}=f_{p} \cup \bigcup_{n \in X} \bigcup_{i=k_{n}}^{n} H_{i, i+k_{n}}$.

Theorem 5.1. It is consistent that $A\left(\mathcal{I}_{1 / x}\right)=\aleph_{1}<2^{\aleph_{0}}$.

Proof. Let $V$ be a model where $2^{\aleph_{0}}>\aleph_{1}$ and let $V^{\prime}$ be obtained from $V$ by adding $\aleph_{1}$ Cohen reals. Choose permutations $\left\{\pi_{\alpha}\right\}_{\alpha \in \omega_{1}}$ such that $\pi_{\alpha}=\bigcup_{p \in G_{\alpha}} f_{p}$ and $G_{\alpha} \subseteq \mathbb{P}\left(\left\{\pi_{\beta}\right\}_{\beta \in \alpha}\right)$ is generic over $V\left[\left\{\pi_{\beta}\right\}_{\beta \in \alpha}\right]$. Using Lemmas 5.2 and 5.3 it follows that $\left\{\pi_{\alpha}\right\}_{\alpha \in \omega_{1}}$ pairwise almost commute. Let $G \supseteq\left\{\pi_{\alpha}\right\}_{\alpha \in \omega_{1}}$ be a maximal almost Abelian subgroup 7 of the subgroup of all $\pi \in \mathbb{S}\left(\mathcal{I}_{1 / x}\right) / \mathbb{F}\left(\mathcal{I}_{1 / x}\right)$ which are first order definable from some finite subset of $\left\{\pi_{\alpha}\right\}_{\alpha \in \omega_{1}}$. To see that $G$ is maximal in $\mathbb{S}\left(\mathcal{I}_{1 / x}\right) / \mathbb{F}\left(\mathcal{I}_{1 / x}\right)$ suppose that $\pi \in V\left[\left\{\pi_{\beta}\right\}_{\beta \in \alpha}\right]$. If $\pi$ is first order definable from some finite subset of $\left\{\pi_{\beta}\right\}_{\beta \in \alpha}$ then either $\pi \in G$ or there is some $\theta \in G$ such that $\{n \in \mathbb{N}: \pi(\theta(n)) \neq \theta(\pi(n))\} \in \mathcal{I}_{1 / x}^{+}$. On the other hand, if $\pi$ is not first order definable from some finite subset of $\left\{\pi_{\beta}\right\}_{\beta \in \alpha}$ then by Lemma 5.4 and genericity it follows that $\left\{n \in \mathbb{N}: \pi\left(\pi_{\alpha}(n)\right) \neq \pi_{\alpha}(\pi(n))\right\} \in \mathcal{I}_{1 / x}^{+}$.

\section{It is POSSIBle that $\mathfrak{a}(\mathcal{I})<A(\mathcal{I})$}

Since it has been shown in Proposition 2.1 that $A\left([\mathbb{N}]^{<\aleph_{0}}\right) \leq \mathfrak{a}$ it is natural to wonder whether there might not be a more general result asserting that $A(\mathcal{I})$ is bounded by $\mathfrak{a}(\mathcal{I})$ as defined in Definition 1.2 . It will be shown that no such result holds, at least not in the generality indicated.

Fix an increasing sequence of integers $\mathcal{N}=\left\{n_{i}\right\}_{i=0}^{\infty}$ such that

$$
\lim _{i \rightarrow \infty} \frac{n_{i+1}-n_{i}}{n_{i+2}-n_{i+1}}=0
$$

and define

$$
\mathcal{I}(\mathcal{N})=\left\{A \subseteq \mathbb{N}: \lim _{i \rightarrow \infty} \frac{\left|A \cap\left[n_{i}, n_{i+1}\right)\right|}{n_{i+1}-n_{i}}=0\right\} .
$$

Theorem 6.1. $A(\mathcal{I}(\mathcal{N}))=2^{\aleph_{0}}$.

Proof. To begin the following claim will be established:

Claim 5. If $g \in \mathbb{S}(\mathcal{I}(\mathcal{N}))$ then there is $B \in \mathcal{I}(\mathcal{N})$ such that if $j \in\left[n_{i}, n_{i+1}\right) \backslash B$ then $g(j) \in\left[n_{i}, n_{i+1}\right)$.

Proof. Let $B^{+}=\bigcup_{i=0}^{\infty}\left\{n_{i} \leq n<n_{i+1}: g(n) \geq n_{i+1}\right\}$ and let $B^{-}=\bigcup_{i=0}^{\infty}\left\{n_{i} \leq n<n_{i+1}: g(n)<n_{i}\right\}$. If $B^{+} \cup B^{-} \in \mathcal{I}(\mathcal{N})$ then the claim is proved. To begin suppose $B^{+} \notin \mathcal{I}(\mathcal{N})$. Choose $\epsilon>0$ and an infinite $Y \subseteq \mathbb{N}$ such that

$$
\frac{\left|B^{+} \cap\left[n_{i}, n_{i+1}\right)\right|}{n_{i+1}-n_{i}} \geq \epsilon
$$

for each $i \in Y$. By thinning out $Y$ it may also be assumed that if $i$ and $j$ belong to $Y$ and $i<j$ then $g\left(B^{+} \cap\left[n_{i}, n_{i+1}\right) \subseteq n_{j}\right.$. It follows that $g\left(B^{+}\right) \cap\left[n_{i+1}, n_{j}\right)=g\left(B^{+} \cap\left[n_{i}, n_{i+1}\right) \cap\left[n_{i+1}, n_{j}\right)\right.$. Therefore, if $i<k<j$ then

$$
\frac{\left|g\left(B^{+}\right) \cap\left[n_{k}, n_{k+1}\right)\right|}{n_{k+1}-n_{k}} \leq \frac{\left.n_{i+1}\right)-n_{i}}{n_{k+1}-n_{k}}
$$

and so $g\left(B^{+}\right) \in \mathcal{I}(\mathcal{N})$ contradicting that $g \in \mathbb{S}(\mathcal{I}(\mathcal{N}))$. A similar argument applied to $g^{-1}$ deals with $B^{-}$.

\footnotetext{
${ }^{7}$ Observe that while the elements of the group generated by $\left\{\pi_{\alpha}\right\}_{\alpha \in \omega_{1}}$ pairwise almost commute the same can not be said of the elements of $G$. All that can be said of them is that they almost commute modulo the ideal $\mathcal{I}_{1 / x}$.
} 
Given a subgroup $G \subseteq \mathbb{S}(\mathcal{I}(\mathcal{N})) / \mathbb{F}(\mathcal{I}(\mathcal{N}))$ let $g \in G$ be different from the identity. Hence $\{n$ : $f(n) \neq n\} \in \mathcal{I}(\mathcal{N})^{+}$and, using the Claim, there is $B \in \mathcal{I}(\mathcal{N})$ such that if $j \in\left[n_{i}, n_{i+1}\right) \backslash B$ then $g(j) \in\left[n_{i}, n_{i+1}\right)$. Let $g^{\prime}$ be a permutation such that $g^{\prime} \uparrow \mathbb{N} \backslash B=g \uparrow \mathbb{N} \backslash B$ and $g^{\prime} \uparrow\left[n_{i}, n_{i+1}\right)$ is a permutation of $\left[n_{i}, n_{i+1}\right)$ for each $i$. (This is possible since $g\left\lceil\left[n_{i}, n_{i+1}\right) \backslash B \rightarrow\left[n_{i}, n_{i+1}\right)\right.$ is one-to-one.) Note that $g^{\prime} \in \mathbb{S}(\mathcal{I}(\mathcal{N})) / \mathbb{F}(\mathcal{I}(\mathcal{N}))$. For any $Z \subseteq \mathbb{N}$ let $g_{Z}$ be defined by

$$
g_{Z}(j)= \begin{cases}g^{\prime}(j) & \text { if } j \in\left[n_{i}, n_{i+1}\right) \text { and } i \in Z \\ j & \text { if } j \in\left[n_{i}, n_{i+1}\right) \text { and } i \notin Z\end{cases}
$$

and note that $g_{Z} \in \mathbb{S}(\mathcal{I}(\mathcal{N})) / \mathbb{F}(\mathcal{I}(\mathcal{N}))$ for each $Z$.

Now, suppose that $h \in \mathbb{S}(\mathcal{I}(\mathcal{N})) / \mathbb{F}(\mathcal{I}(\mathcal{N}))$ and use the claim to find $C \in \mathcal{I}(\mathcal{N})$ such that $j \in$ $\left[n_{i}, n_{i+1}\right) \backslash B$ then $g(j) \in\left[n_{i}, n_{i+1}\right)$. Let $D$ be such that $h(g(j))=g(h(j))$ for $j \in \mathbb{N} \backslash D$. Then let $E=C \cup h(B) \cup h^{-1}(B) \cup D$ and note that $E \in \mathcal{I}(\mathcal{N})$ since $h \in \mathbb{S}(\mathcal{I}(\mathcal{N})) / \mathbb{F}(\mathcal{I}(\mathcal{N}))$. Now observe that $g_{Z}(h(j))=h\left(g_{Z}(j)\right)$ for each $j \in \mathbb{N} \backslash E$. To see this let $j \in\left[n_{i}, n_{i+1}\right)$ and suppose first that $i \in Z$. In this case $g_{Z}(j)=g^{\prime}(j)=g(j)$. Furthermore, since $j \notin C, h(j) \in\left[n_{i}, n_{i+1}\right)$ and hence $g(h(j))=g_{Z}(h(j)$. Since $j \notin D$ it follows that $h(g(j))=g(h(j)$ and, hence, In this case $h\left(g_{Z}(j)\right)=g_{Z}(h(j))$. If $i \notin Z$ then $g_{Z}(j)=j$ and, since $j \notin D, h(j) \in\left[n_{i}, n_{i+1}\right)$. Therefore, $g_{Z}(h(j))=h(j)$ and so $g_{Z}(h(j))=h\left(g_{Z}(j)\right)$.

Theorem 6.2. $\mathfrak{a}(\mathcal{I}(\mathcal{N})) \leq \mathfrak{a}$.

Proof. Let $\mathcal{A}$ be a maximal almost disjoint family of size a. For $A \in \mathcal{A}$ define $A^{*}=\bigcup_{i \in A}\left[n_{i}, n_{i+1}\right)$ and let $\mathcal{A}^{*}=\left\{A^{*}: A \in \mathcal{A}\right\}$. Then $\mathcal{A}^{*}$ is maximal in $\mathcal{P}(\mathbb{N}) / \mathcal{I}(\mathcal{N})$.

\section{Questions}

Question 7.1. Can the lower bound $A(\mathbb{S} / \mathbb{F}) \geq \mathfrak{p}$ of Theorem 3.2 be improved?

For any function $h: \mathbb{N} \rightarrow \mathbb{R}$ one can define the summable ideal $\mathcal{I}_{h}$ to be the set of all $X \subseteq \mathbb{N}$ such that $\sum_{x \in X} h(x)<\infty$. Observe that it is possible to modify the proof of Theorem 6.1 in order to replace the ideal $\mathcal{I}(\mathcal{N})$ by a summable ideal. In particular, let $\left\{n_{i}\right\}_{i=0}^{\infty}$ be an increasing sequence of integers defined by $n_{i+1}-n_{i}=n_{i}^{3}$ and let $h$ be defined by $h(j)=n_{i}^{-3}$ if $n_{i} \leq j<n_{i+1}$. If $g \in \mathbb{S}\left(\mathcal{I}_{h}\right)$ and $B^{+}$and $B^{-}$are defined as in the proof of Theorem 6.1 then it is easy to see that $\sum_{j \in B^{+} \cap n_{i}} h(g(j)) \leq\left|B^{+} \cap n_{i}\right| n_{i}^{-3} \leq n_{i}^{-2}$ and hence Claim 5 still holds as does the remainder of the argument of Theorem 6.1. Hence $A\left(\mathcal{I}_{h}\right)=2^{\aleph_{0}}$. This motivates the following question.

Question 7.2. For which functions $h$ is it possible to improve Theorem 5.1 to show that $A\left(\mathcal{I}_{h}\right)=$ $\aleph_{1}<2^{\aleph_{0}}$ in the model obtained by adding $\aleph_{1}$ Cohen reals?

Question 7.3. Are there functions $h$ and $g$ such that it is consistent that $A\left(\mathcal{I}_{h}\right)<A\left(\mathcal{I}_{g}\right)<2^{\aleph_{0}}$ ?

Question 7.4. Is it possible to characterize the summable ideals $\mathcal{I}_{h}$ such that $A\left(\mathcal{I}_{h}\right)=2^{\aleph_{0}}$ ? Can the same be done for the $F_{\sigma}$ ideals or all analytic ideals?

Question 7.5. Can Theorem 5.1 be improved to show that it is consistent with set theory that $2^{\aleph_{0}}>\aleph_{1}$ yet there is an almost commuting subgroup of $\mathbb{S}$ of cardinality $\aleph_{1}$ which is maximal with respect to commuting modulo $\mathcal{I}_{1 / x}$ ? Does this hold in the Cohen model of Theorem 5.1]

The methods of Sections 4 and 5 require that the subgroups constructed contain many involutions. While the methods can be modified to produce groups with no elements of order $k$ for a fixed $k$, the following questions seem more subtle.

Question 7.6. Can Theorem 4.1 be modified to assert that it is consistent that there is a maximal, Abelian, torsion free subgroup of $\mathbb{S} / \mathbb{F}$ of size $\aleph_{1}$ and $\aleph_{1}<\mathfrak{a}$ ?

Question 7.7. Can Theorem 5.1 be modified to assert that it is consistent that there is a maximal, Abelian, torsion free subgroup of $\mathbb{S}\left(\mathcal{I}_{1 / x}\right) / \mathbb{F}\left(\mathcal{I}_{1 / x}\right)$ of size $\aleph_{1}$ and $\aleph_{1}<2^{\aleph_{0}}$ ? 


\section{REFERENCES}

[1] J. L. Alperin, Jacinta Covington, and Dugald Macpherson. Automorphisms of quotients of symmetric groups. In Ordered groups and infinite permutation groups, pages 231-247. Kluwer Acad. Publ., Dordrecht, 1996.

[2] Tomek Bartoszyński and Haim Judah. Set Theory - On the structure of the real line. A K Peters, 1995.

[3] Murray G. Bell. On the combinatorial principle P(c). Fund. Math., 114(2):149-157, 1981.

[4] J. M. Burns and B. Goldsmith. Maximal order abelian subgroups of symmetric groups. Bull. London Math. Soc., 21(1):70-72, 1989.

[5] Ilijas Farah. Analytic quotients: theory of liftings for quotients over analytic ideals on the integers. Mem. Amer. Math. Soc., 148(702):xvi+177, 2000.

[6] Richard Laver. On the consistency of Borel's conjecture. Acta Math., 137(3-4):151-169, 1976.

[7] Jan Mycielski. Some theorems on connected compact groups. Bull. Acad. Polon. Sci. Cl. III, 5:1023, LXXXV, 1957.

[8] Jan Mycielski. Some properties of connected compact groups. Colloq. Math., 5:162-166, 1958.

[9] Walter Rudin. Homogeneity problems in the theory of Čech compactifications. Duke Math. J., 23:409-419, 1956.

[10] J. Schreier and S. Ulam. Uber die permutationsgruppe der nat"urlichen zahlenfolge. Studia Math., 14:134-141, 1933.

[11] S. Shelah and J. K. Truss. On distinguishing quotients of symmetric groups. Ann. Pure Appl. Logic, 97(1-3):47-83, 1999.

[12] Saharon Shelah. Non-elementary proper forcing notions. Shelah [Sh:630].

[13] Saharon Shelah. First order theory of permutation groups. Israel J. Math., 14:149-162, 1973.

[14] J. Steprāns. The almost disjointness cardinal invariant in the quotient algebra of the rationals modulo the nowhere dense subsets. http://hausdorff.math.yorku.ca/Research/menu.html, 2001.

[15] Reinhard Winkler. On maximal abelian groups of maps. J. Austral. Math. Soc. Ser. A, 55(3):414-420, 1993.

Department of Mathematics, Rutgers University, Hill Center, Piscataway, New Jersey, U.S.A. 088548019

Current address: Institute of Mathematics, Hebrew University, Givat Ram, Jerusalem 91904, Israel

E-mail address: shelah@math.rutgers.edu

Department of Mathematics, York University, 4700 Keele Street, North York, Ontario, Canada M3J $1 \mathrm{P} 3$

E-mail address: steprans@yorku.ca 